Article

\title{
Dual Generalized Nonnegative Normal Neutrosophic Bonferroni Mean Operators and Their Application in Multiple Attribute Decision Making
}

\author{
Jiongmei Mo and Han-Liang Huang * \\ School of Mathematics and Statistics, Minnan Normal University, Zhangzhou 363000, Fujian, China; \\ mojiongmei123@126.com \\ * Correspondence: huanghl@mnnu.edu.cn
}

Received: 19 July 2018; Accepted: 27 July 2018; Published: 6 August 2018

\begin{abstract}
For multiple attribute decision making, ranking and information aggregation problems are increasingly receiving attention. In a normal neutrosophic number, the ranking method does not satisfy the ranking principle. Moreover, the proposed operators do not take into account the correlation between any aggregation arguments. In order to overcome the deficiencies of the existing ranking method, based on the nonnegative normal neutrosophic number, this paper redefines the score function, the accuracy function, and partial operational laws. Considering the correlation between any aggregation arguments, the dual generalized nonnegative normal neutrosophic weighted Bonferroni mean operator and dual generalized nonnegative normal neutrosophic weighted geometric Bonferroni mean operator were investigated, and their properties are presented. Here, these two operators are applied to deal with a multiple attribute decision making problem. Example results show that the proposed method is effective and superior.
\end{abstract}

Keywords: multiple attribute decision making; nonnegative normal neutrosophic number; aggregation operator

\section{Introduction}

During the decision making process, the evaluation information given by decision makers is often incomplete, indeterminate, and inconsistent. To deal with this uncertain information, fuzzy set (FS) was proposed by Zadeh [1] in 1965. On the basis of FS, intuitionistic fuzzy set (IFS) was introduced by Atanassov [2] in 1986. However, IFS can not deal with all types of indeterminate and inconsistent information. Hence, considering the indeterminacy-membership based on IFS, Smarandache [3] developed the neutrosophic set (NS) in 1995. In NS, the truth-membership function, indeterminacy-membership function, and false-membership function are independent of each other. In real life, normal distribution is widely applied. Nevertheless, FS, IFS, and NS do not take the normal distribution into account. Therefore, the normal fuzzy number (NFN) was firstly introduced by Yang and Ko [4] in 1996, and NFN can deal with normal fuzzy information. Based on IFS and NFN, normal intuitionistic fuzzy number (NIFN) was defined by Wang and Li [5] in 2002. Further, combining NFN with NS, Liu [6] proposed the normal neutrosophic number (NNN).

With the development of society, many achievements have been made in the research of multiple attribute decision making (MADM) [7-10]. Chatterjee et al. [7] proposed a novel hybrid method encompassing factor relationship (FARE) and multi-attributive border approximation area comparison (MABAC) methods. Petković et al. [8] introduced the performance selection index (PSI) method for solving machining MADM problems. Roy et al. [9] developed a rough strength relational-decision making and trial evaluation laboratory model. Badi et al. [10] used a new combinative distance-based assessment (CODAS) method to handle MADM problems. Lee et al. [11] developed fuzzy entropy, 
which determined for an FS, by using the distance measure. Based on IFS, some authors [12-14] investigated the distance-based technique for order preference by similarity to an ideal solution (TOPSIS)method, entropy, and similarity measures of IFS, and applied them to MADM. Atanassov and Gargov [15] extended IFS to interval valued intuitionistic fuzzy set (IVIFS). Huang [16] proposed a (T, S)-based IVIF composition matrix and its application. Chen et al. [17] and Biswas et al. [18] introduced a linear programming methodology and integrated the TOPSIS approach for MADM in IVIFS. Because NS-a generalization of IFS and FS - can better describe uncertain information, NS now attracts great attention. An outranking method, COPRAS method, and entropy with NS for MADM has been developed in [19-21]. Wang et al. [22,23] introduced interval neutrosophic set (INS) and single-valued neutrosophic set (SVNN). Zhang et al. [24,25] and Tian et al. [26] proposed an outranking approach and weighted correlation coefficient: cross-entropy with INS for MADM. Further, Huang [27] and Ye [28] presented a new distance measure-cross-entropy and its application to MADM in SVNN.

Presently, information aggregation operators are attracting an increasing amount of attention for dealing with MADM. Many aggregation operators have been developed in intuitionistic fuzzy MADM [29-31]. Wang and Li [32,33] and Wang et al. [34] developed some intuitionistic normal aggregation operators and proposed some MADM methods based on these operators, while Wang et al. [5] developed some aggregation operators for NIFN. For NS and INS, some aggregation operators were proposed, such as power aggregation operators [35], generalized weighted power averaging operator [36], order weighted aggregation operators [37], generalized weighted power averaging operator [38], etc. Liu [39-41] developed Frank operators, generalized weighted power averaging operators, and Heronian mean operators for application with NNN; Şahin [42] introduced generalized prioritized aggregation operators with NNN.

However, these operators do not consider the relationship between attributes. Considering the interrelation between attributes, Bonferroni mean (BM) operator was first defined by Bonferroni [43]. Liu [6] introduced normal neutrosophic weighted Bonferroni mean (NNWBM) operator and normal neutrosophic weighted geometric Bonferroni mean (NNWGBM) operator. However, these operators only take into account correlations between any two aggregation arguments, and they do not consider the connections among any three or more than three aggregation arguments. The score function and accuracy function of the NNN and their ranking method were proposed also. However, the score function and accuracy function do not satisfy the ranking principle and are counterintuitive (see Example 1 for details).

The main contribution of this paper is (1) the proposal of a score function and accuracy function that satisfy the ranking principle, and (2) the extension of the operators in Liu [6]. First, we introduce the nonnegative normal neutrosophic number (NNNN). Then, a new score function and accuracy function are defined to solve the problem of the original function. Furthermore, considering the connections between any two or more than two aggregation arguments, the operator in [6] is generalized and some new operators are defined. For MADM, it is more reasonable to consider the relationship between each attribute, and the example in this paper further illustrates the advantages of the proposed MADM method compared with Liu [6]. The example in this paper further shows that when the relationship between more aggregation arguments is considered, the aggregation result is more stable; when the parameter value is larger, the aggregation result is more sensitive.

The structure of this paper as follows. Section 2 reviews the NNN, some operational laws, the score function, accuracy function, and the ranking method. Section 3 proposes the basic concept of the NNNN, and the new score function and accuracy function are introduced. Some generalized aggregation operators are developed, which are the dual generalized nonnegative normal neutrosophic weighted Bonferroni mean (DGNNNWBM) operator and dual generalized nonnegative normal neutrosophic weighted geometric Bonferroni mean (DGNNNWGBM) operator. Their properties are discussed. In Section 4, based on the DGNNNWBM operator and DGNNNWGBM operator, a MADM method is 
established. Section 5 gives a numerical example to explain the application of the proposed MADM method, and compares it with the method presented in [6]. Section 6 concludes this paper.

\section{Preliminaries}

Yang and Ko (1996) introduced the concept of the normal fuzzy number (NFN).

Definition 1. [4] $A=(a, \sigma)$ is an NFN if its membership function is defined by:

$$
A(x)=e^{-\left(\frac{x-a}{\sigma}\right)^{2}}(x \in X, \sigma>0) \text {, }
$$

where $X$ is the set of real numbers, and the set of NFNs is denoted as $\tilde{N}$.

The neutrosophic number (NN) and single-valued neutrosophic number (SVNN) were proposed in 1995 and 2005.

Definition 2. [3] Let $X$ be a universe of discourse, with a generic element in $X$ denoted by $x$. An NN $A$ in $X$ is

$$
A(x)=\left\langle x \mid\left(T_{A}(x), I_{A}(x), F_{A}(x)\right)\right\rangle,
$$

where $T_{A}(x)$ denotes the truth-membership function, $I_{A}(x)$ denotes the indeterminacy-membership function, and $F_{A}(x)$ denotes the falsity-membership function. $T_{A}(x), I_{A}(x)$, and $F_{A}(x)$ are real standard or nonstandard subsets of $]^{-} 0,1^{+}$.

There is no limitation on the sum of $T_{A}(x), I_{A}(x)$, and $F_{A}(x)$, so ${ }^{-} 0 \leq T_{A}(x)+I_{A}(x)+F_{A}(x) \leq 3^{+}$.

Definition 3. [23] Let X be a universe of discourse, with a generic element in X denoted by $x$. An SVNN A in $\mathrm{X}$ is depicted by the following:

$$
A(x)=\left\langle x \mid\left(T_{A}(x), I_{A}(x), F_{A}(x)\right)\right\rangle,
$$

where $T_{A}(x)$ denotes the truth-membership function, $I_{A}(x)$ denotes the indeterminacy-membership function, and $F_{A}(x)$ denotes the falsity-membership function. For each point $x$ in $X$, we have $T_{A}(x), I_{A}(x), F_{A}(x) \in[0,1]$ and $0 \leq T_{A}(x)+I_{A}(x)+F_{A}(x) \leq 3$.

Based on NFN and NN, Liu (2017) defined the normal neutrosophic number (NNN).

Definition 4. [6] Let $X$ be a universe of discourse, with a generic element in $X$ denoted by $x$, and $(a, \sigma) \in \tilde{N}$; then, an NNN $A$ in $X$ is expressed as:

$$
A(x)=\left\langle x \mid(a, \sigma),\left(T_{A}(x), I_{A}(x), F_{A}(x)\right)\right\rangle, x \in X,
$$

where the truth-membership function $T_{A}(x)$ satisfies:

$$
T_{A}(x)=T_{A} e^{-\left(\frac{x-a}{\sigma}\right)^{2}}, x \in X,
$$

where the indeterminacy-membership function $I_{A}(x)$ satisfies:

where the falsity-membership function $F_{A}(x)$ satisfies:

$$
I_{A}(x)=1-\left(1-I_{A}\right) e^{-\left(\frac{x-a}{\sigma}\right)^{2}}, x \in X,
$$

$$
F_{A}(x)=1-\left(1-F_{A}\right) e^{-\left(\frac{x-a}{\sigma}\right)^{2}}, x \in X .
$$

For each point $x$ in $X$, we have $T_{A}(x), I_{A}(x), F_{A}(x) \in[0,1]$, and $0 \leq T_{A}(x)+I_{A}(x)+F_{A}(x) \leq 3$. Then, we denote $\tilde{a}=\langle(a, \sigma),(T, I, F)\rangle$ as an NNN.

Some operational laws are shown in the following.

Definition 5. [6] Let $\tilde{a}_{1}=\left\langle\left(a_{1}, \sigma_{1}\right),\left(T_{1}, I_{1}, F_{1}\right)\right\rangle$ and $\tilde{a}_{2}=\left\langle\left(a_{2}, \sigma_{2}\right),\left(T_{2}, I_{2}, F_{2}\right)\right\rangle$ be two NNNs; then, the operational rules are defined as follows:

(1) $\tilde{a}_{1} \oplus \tilde{a}_{2}=\left\langle\left(a_{1}+a_{2}, \sigma_{1}+\sigma_{2}\right),\left(T_{1}+T_{2}-T_{1} T_{2}, I_{1} I_{2}, F_{1} F_{2}\right)\right\rangle$;

(2) $\tilde{a}_{1} \otimes \tilde{a}_{2}=\left\langle\left(a_{1} a_{2}, a_{1} a_{2} \sqrt{\frac{\sigma_{1}^{2}}{a_{1}^{2}}+\frac{\sigma_{2}^{2}}{a_{2}^{2}}}\right),\left(T_{1} T_{2}, I_{1}+I_{2}-I_{1} I_{2}, F_{1}+F_{2}-F_{1} F_{2}\right)\right\rangle$;

(3) $\lambda \tilde{a}_{1}=\left\langle\left(\lambda a_{1}, \lambda \sigma_{1}\right),\left(1-\left(1-T_{1}\right)^{\lambda}, I_{1}^{\lambda}, F_{1}^{\lambda}\right)\right\rangle(\lambda>0)$;

(4) $\tilde{a}_{1}^{\lambda}=\left\langle\left(a_{1}^{\lambda}, \lambda^{\frac{1}{2}} a_{1}^{\lambda-1} \sigma_{1}\right),\left(T_{1}^{\lambda}, 1-\left(1-I_{1}\right)^{\lambda}, 1-\left(1-F_{1}\right)^{\lambda}\right)\right\rangle(\lambda>0)$. 
Theorem 1. [6] Let $\tilde{a}_{1}=\left\langle\left(a_{1}, \sigma_{1}\right),\left(T_{1}, I_{1}, F_{1}\right)\right\rangle$ and $\tilde{a}_{2}=\left\langle\left(a_{2}, \sigma_{2}\right),\left(T_{2}, I_{2}, F_{2}\right)\right\rangle$ be two NNNs, and $\eta, \eta_{1}, \eta_{2}>0$; then, we have

(1) $\tilde{a}_{1} \oplus \tilde{a}_{2}=\tilde{a}_{2} \oplus \tilde{a}_{1}$

(2) $\tilde{a}_{1} \otimes \tilde{a}_{2}=\tilde{a}_{2} \otimes \tilde{a}_{1}$

(3) $\eta\left(\tilde{a}_{1} \oplus \tilde{a}_{2}\right)=\eta \tilde{a}_{1} \oplus \eta \tilde{a}_{2}$;

(4) $\eta_{1} \tilde{a}_{1} \oplus \eta_{2} \tilde{a}_{1}=\left(\eta_{1}+\eta_{2}\right) \tilde{a}_{1}$;

(5) $\tilde{a}_{1}^{\eta} \otimes \tilde{a}_{2}^{\eta}=\left(\tilde{a}_{1} \otimes \tilde{a}_{2}\right)^{\eta}$;

(6) $\tilde{a}_{1}^{\eta_{1}} \otimes \tilde{a}_{1}^{\eta_{2}}=\tilde{a}_{1}^{\eta_{1}+\eta_{2}}$.

Liu (2017) proposed the score function and accuracy function for an NNN.

Definition 6. [41] Let $\tilde{a}_{k}=\left\langle\left(a_{k}, \sigma_{k}\right),\left(T_{k}, I_{k}, F_{k}\right)\right\rangle$ be an $N N N$, then its score function is

$$
\begin{aligned}
& s_{1}\left(\tilde{a}_{k}\right)=a_{k}\left(2+T_{k}-I_{k}-F_{k}\right), \\
& s_{2}\left(\tilde{a}_{k}\right)=\sigma_{k}\left(2+T_{k}-I_{k}-F_{k}\right) ;
\end{aligned}
$$

and its accuracy function is

$$
\begin{aligned}
& h_{1}\left(\tilde{a}_{k}\right)=a_{k}\left(2+T_{k}-I_{k}+F_{k}\right), \\
& h_{2}\left(\tilde{a}_{k}\right)=\sigma_{k}\left(2+T_{k}-I_{k}+F_{k}\right) .
\end{aligned}
$$

Zhang et al. (2017) proposed the dual generalized weighted Bonferroni mean (DGWBM) operator and dual generalized weighted geometric Bonferroni mean (DGWGBM) operator.

Definition 7. [44] Let $a_{i}(i=1,2, \ldots, n)$ be a collection of nonnegative crisp numbers with the weight $\omega=$ $\left(\omega_{1}, \omega_{2}, \ldots, \omega_{n}\right)^{T}, \omega_{i} \in[0,1](i=1,2, \ldots, n)$ and $\sum_{i=1}^{n} \omega_{i}=1$. If

$$
\operatorname{DGWBM}^{R}\left(a_{1}, a_{2}, \ldots, a_{n}\right)=\left(\sum_{i_{1}, i_{2}, \ldots, i_{n}=1}^{n}\left(\prod_{j=1}^{n} \omega_{i_{j}} a_{i_{j}}^{r_{j}}\right)\right)^{1 / \sum_{j=1}^{n} r_{j}},
$$

where $R=\left(r_{1}, r_{2}, \ldots, r_{n}\right)^{T}$ is the parameter vector with $r_{j} \geq 0(i=1,2, \ldots, n)$.

Definition 8. [44] Let $a_{i}(i=1,2, \ldots, n)$ be a collection of nonnegative crisp numbers with the weight $\omega=$ $\left(\omega_{1}, \omega_{2}, \ldots, \omega_{n}\right)^{T}$, where $\omega_{i} \in[0,1](i=1,2, \ldots, n)$ and $\sum_{i=1}^{n} \omega_{i}=1$. if

$$
\operatorname{DGWBM}^{R}\left(a_{1}, a_{2}, \ldots, a_{n}\right)=\frac{1}{\sum_{j=1}^{n} r_{j}}\left(\prod_{i_{1}, i_{2}, \ldots, i_{n}}^{n}\left(\sum_{j=1}^{n}\left(r_{j} a_{i_{j}}\right)\right)\right)^{\prod_{j=1}^{n} \omega_{i_{j}}},
$$

where $R=\left(r_{1}, r_{2}, \ldots, r_{n}\right)^{T}$ is the parameter vector with $r_{i} \geq 0(i=1,2, \ldots, n)$.

\section{Main Results}

\subsection{Ranking of Nonnegative Normal Neutrosophic Number}

Liu and Li (2017) [6] introduced the concept of the score function $s_{1}$ and $s_{2}$, and the accuracy function $h_{1}$ and $h_{2}$, as shown in Definition 6. We found some deficiencies with the ranking of these functions, as shown below.

Let $\tilde{a}_{k}=\left\langle\left(a_{k}, \sigma_{k}\right),\left(T_{k}, I_{k}, F_{k}\right)\right\rangle(k=1,2)$ be any two NNNs. When $T_{1}<T_{2}, I_{1}>I_{2}, F_{1}>F_{2}$, $a_{1} \leq a_{2}$, and $\sigma_{1} \geq \sigma_{2}$ :

(1) If $a_{k}>0$ or $a_{k}<0$, then ranking results may be completely opposite;

(2) When $s_{1}$ can determine the ranking result of $a_{k}$, the influence of $\sigma_{k}$ is not considered;

(3) Neither the score function nor the accuracy function satisfy the monotonicity.

We use the following example to illustrate problems (1) and (3) mentioned above.

Example 1. Let $\tilde{a}_{1}$ and $\tilde{a}_{2}$ be two NNNs, where the specific values are as shown in Table 1. According to

$$
\begin{aligned}
& s_{1}\left(\tilde{a}_{k}\right)=a_{k}\left(2+T_{k}-I_{k}-F_{k}\right), \\
& s_{2}\left(\tilde{a}_{k}\right)=\sigma_{k}\left(2+T_{k}-I_{k}-F_{k}\right),
\end{aligned}
$$




$$
\begin{aligned}
& h_{1}\left(\tilde{a}_{k}\right)=a_{k}\left(2+T_{k}-I_{k}+F_{k}\right), \\
& h_{2}\left(\tilde{a}_{k}\right)=\sigma_{k}\left(2+T_{k}-I_{k}+F_{k}\right),
\end{aligned}
$$

we can get its score function and accuracy function from Table 1. For number 1 ,

$$
s_{1}\left(\tilde{a}_{1}\right)=1 \times(2+0.5-0.2-0.2)=2.1, s_{1}\left(\tilde{a}_{2}\right)=2 \times(2+0.6-0.1-0.1)=4.8,
$$

by $2.1<4.8$, we have $\tilde{a}_{1}<\tilde{a}_{2}$. For number 2 ,

$$
s_{1}\left(\tilde{a}_{1}\right)=(-1) \times(2+0.5-0.2-0.2)=-2.1, s_{1}\left(\tilde{a}_{2}\right)=(-0.95) \times(2+0.6-0.1-0.1)=-2.28,
$$

by $-2.1>-2.28$, we have $\tilde{a}_{1}>\tilde{a}_{2}$ for the numerical results, which are shown in Table 2.

From Table 2, when $T_{1}<T_{2}, I_{1}>I_{2}, F_{1}>F_{2}, a_{1} \leq a_{2}$, and $\sigma_{1} \geq \sigma_{2}$ are satisfied, we can intuitively see that the score function and accuracy function will be ranked differently if different values are taken. For example, the number 1 satisfies $0.5<0.6,0.2>0.1,0.2>0.1,1<2,0.3>0.1$; the number 2 satisfies $0.5<0.6,0.2>0.1$, $0.2>0.1,-1<-0.95,0.2>0.1$. However, their ranking results are completely different. The ranking results of numbers 2, 4, 6, 8 in Table 2 are counterintuitive. For example, the number 2 satisfies $0.5<0.6,0.2>0.1,0.2\rangle$ $0.1,-1<-0.95,0.2>0.1$, and the ranking result are $\tilde{a}_{1}>\tilde{a}_{2}$. However, intuitively, $\tilde{a}_{2}$ should be ranked first.

Table 1. The numerical example.

\begin{tabular}{ccc}
\hline Number & $\tilde{\boldsymbol{a}}_{\mathbf{1}}$ & $\tilde{\boldsymbol{a}}_{\mathbf{2}}$ \\
\hline 1 & $\langle(1,0.3),(0.5,0.2,0.2)\rangle$ & $\langle(2,0.1),(0.6,0.1,0.1)\rangle$ \\
2 & $\langle(-1,0.2),(0.5,0.2,0.2)\rangle$ & $\langle(-0.95,0.1),(0.6,0.1,0.1)\rangle$ \\
3 & $\langle(2,0.4),(0.6,0.2,0.3)\rangle$ & $\langle(2.5,0.2),(0.7,0.1,0.1)\rangle$ \\
4 & $\langle(2,0.4),(0.6,0.2,1)\rangle$ & $\langle(2.5,0.2),(0.7,0.1,0.1)\rangle$ \\
5 & $\langle(0,1),(0.6,0.3,0.2)\rangle$ & $\langle(0,0.5),(0.7,0.05,0.05)\rangle$ \\
6 & $\langle(0,1),(0.6,0.3,0.2)\rangle$ & $\langle(0,0.9),(0.7,0.05,0.05)\rangle$ \\
7 & $\langle(2,2),(0.6,0.8,0.2)\rangle$ & $\langle(2,1),(0.7,0,0.1)\rangle$ \\
8 & $\langle(2,2),(0.6,0.8,0.2)\rangle$ & $\langle(2,1.5),(0.7,0,0.1)\rangle$ \\
\hline
\end{tabular}

Table 2. The score function and accuracy function of the numerical example.

\begin{tabular}{cccccccccc}
\hline Number & $\boldsymbol{s}_{\mathbf{1}}\left(\tilde{a}_{\mathbf{1}}\right)$ & $\boldsymbol{s}_{\mathbf{1}}\left(\tilde{a}_{\mathbf{2}}\right)$ & $\boldsymbol{h}_{\mathbf{1}}\left(\tilde{\boldsymbol{a}}_{\mathbf{1}}\right)$ & $\boldsymbol{h}_{\mathbf{1}}\left(\tilde{a}_{\mathbf{2}}\right)$ & $\boldsymbol{s}_{\mathbf{2}}\left(\tilde{a}_{\mathbf{1}}\right)$ & $\boldsymbol{s}_{\mathbf{2}}\left(\tilde{\boldsymbol{a}}_{\mathbf{2}}\right)$ & $\boldsymbol{h}_{\mathbf{2}}\left(\tilde{a}_{\mathbf{1}}\right)$ & $\boldsymbol{h}_{\mathbf{2}}\left(\tilde{a}_{\mathbf{2}}\right)$ & Ranking \\
\hline 1 & 2.1 & 4.8 & - & - & - & - & - & - & $\tilde{a}_{1}<\tilde{a}_{2}$ \\
2 & -2.1 & -2.28 & - & - & - & - & - & - & $\tilde{a}_{1}>\tilde{a}_{2}$ \\
3 & - & - & 5.4 & 6.75 & - & - & - & - & $\tilde{a}_{1}<\tilde{a}_{2}$ \\
4 & - & - & 6.8 & 6.75 & - & - & - & - & $\tilde{a}_{1}>\tilde{a}_{2}$ \\
5 & - & - & - & - & 2.1 & 1.3 & - & - & $\tilde{a}_{1}<\tilde{a}_{2}$ \\
6 & - & - & - & - & 2.1 & 2.34 & - & - & $\tilde{a}_{1}>\tilde{a}_{2}$ \\
7 & - & - & - & - & - & - & 4 & 2.8 & $\tilde{a}_{1}<\tilde{a}_{2}$ \\
8 & - & - & - & - & - & - & 4 & 4.2 & $\tilde{a}_{1}>\tilde{a}_{2}$ \\
\hline
\end{tabular}

In order to avoid the disadvantages of the ranking, we propose the nonnegative normal neutrosophic number (NNNN). Additionally, we take $\sigma$ into account and introduce the score function and accuracy function of the NNNN.

Definition 9. $A(x)=\left\langle x \mid(a, \sigma),\left(T_{A}(x), I_{A}(x), F_{A}(x)\right)\right\rangle$ is an NNNN if it has satisfied Definition 4 and $a \geq 0$.

Based on the NNNN, the new score function $S$ and accuracy function $H$ are proposed.

Definition 10. Suppose $\tilde{a}=\langle(a, \sigma),(T, I, F)\rangle$ is an NNNN, then its score function is

and its accuracy function is

$$
S(\tilde{a})=\left(a+\frac{1}{\sigma}\right)(2+T-I-F)
$$

$$
H(\tilde{a})=\left(a+\frac{1}{\sigma}\right)(1+T-F) .
$$

According to the score function and accuracy function, the following propositions are derived. 
Proposition 1. Let $\tilde{a}_{k}=\left\langle\left(a_{k}, \sigma_{k}\right),\left(T_{k}, I_{k}, F_{k}\right)\right\rangle(k=1,2)$ be any two NNNNs, then the following conclusions are obtained.

(1) If $a_{1} \leq a_{2}, \sigma_{1} \geq \sigma_{2}, T_{1}<T_{2}$ and $I_{1}>I_{2}$ and $F_{1}>F_{2}$, then $S\left(\tilde{a}_{1}\right)<S\left(\tilde{a}_{2}\right)$;

(2) If $a_{1} \leq a_{2}, \sigma_{1} \geq \sigma_{2}, T_{1}<T_{2}$, and $F_{1}>F_{2}$, then $H\left(\tilde{a}_{1}\right)<H\left(\tilde{a}_{2}\right)$.

Therefore, we have the following ranking principles.

Definition 11. Let $\tilde{a}_{k}=\left\langle\left(a_{k}, \sigma_{k}\right),\left(T_{k}, I_{k}, F_{k}\right)\right\rangle(k=1,2)$ be any two NNNNs, then we have the following method for ranking an $N N N N$ :

(1) If $S\left(\tilde{a}_{1}\right)<S\left(\tilde{a}_{2}\right)$, then $\tilde{a}_{1}<\tilde{a}_{2}$;

(2) If $S\left(\tilde{a}_{1}\right)=S\left(\tilde{a}_{2}\right)$, then

(a) If $H\left(\tilde{a}_{1}\right)<H\left(\tilde{a}_{2}\right)$, then $\tilde{a}_{1}<\tilde{a}_{2}$;

(b) If $H\left(\tilde{a}_{1}\right)=H\left(\tilde{a}_{2}\right)$, then $\tilde{a}_{1} \sim \tilde{a}_{2}$.

We introduce some operational laws as follows:

Definition 12. Let $\tilde{a}_{k}=\left\langle\left(a_{k}, \sigma_{k}\right),\left(T_{k}, I_{k}, F_{k}\right)\right\rangle(k=1,2)$ be any two NNNNs, then the operational rules are defined as follows:

(1) $\tilde{a}_{1} \hat{\otimes} \tilde{a}_{2}=\left\langle\left(a_{1} a_{2}, \sigma_{1} \sigma_{2}\right),\left(T_{1} T_{2}, I_{1}+I_{2}-I_{1} I_{2}, F_{1}+F_{2}-F_{1} F_{2}\right)\right\rangle$;

(2) $\tilde{a}_{1}^{* \lambda}=\left\langle\left(a_{1}^{\lambda}, \sigma_{1}^{\lambda}\right),\left(T_{1}^{\lambda}, 1-\left(1-I_{1}\right)^{\lambda}, 1-\left(1-F_{1}\right)^{\lambda}\right)\right\rangle(\lambda>0)$.

Moreover, the relations of the operational laws are given as below, and these properties are obvious.

Proposition 2. Let $\tilde{a}_{1}=\left\langle\left(a_{1}, \sigma_{1}\right),\left(T_{1}, I_{1}, F_{1}\right)\right\rangle$ and $\tilde{a}_{2}=\left\langle\left(a_{2}, \sigma_{2}\right),\left(T_{2}, I_{2}, F_{2}\right)\right\rangle$ be two NNNNs, and $\eta, \eta_{1}, \eta_{2}>0$; then

(1) $\tilde{a}_{1} \hat{\otimes} \tilde{a}_{2}=\tilde{a}_{2} \hat{\otimes} \tilde{a}_{1}$

(2) $\tilde{a}_{1}^{* \eta} \hat{\otimes} \tilde{a}_{2}^{* \eta}=\left(\tilde{a}_{1} \hat{\otimes} \tilde{a}_{2}\right)^{* \eta}$;

(3) $\tilde{a}_{1}^{* \eta_{1}} \hat{\otimes} \tilde{a}_{1}^{* \eta_{2}}=\tilde{a}_{1}^{*\left(\eta_{1}+\eta_{2}\right)}$.

\subsection{DGNNNWBM Operator and DGNNNWGBM Operator}

This section extends the DGWBM and DGWGBM to NNNN, and proposes the dual generalized nonnegative normal neutrosophic weighted Bonferroni mean (DGNNNWBM) operator and dual generalized nonnegative normal neutrosophic weighted geometric Bonferroni mean (DGNNNWGBM) operator.

Definition 13. Suppose $\left\{\tilde{a}_{i} \mid \tilde{a}_{i}=\left\langle\left(a_{i}, \sigma_{i}\right),\left(T_{i}, I_{i}, F_{i}\right)\right\rangle, i=1,2, \ldots, n\right\}$ is a set of NNNNs, with their weight vector being $\omega_{i}=\left(\omega_{1}, \omega_{2}, \ldots, \omega_{n}\right)^{T}$, where $\omega_{i} \in[0,1]$ and $\sum_{i=1}^{n} \omega_{i}=1$. The DGNNNWBM operator is defined as

$$
\operatorname{DGNNNWBM} M_{\omega}^{R}\left(\tilde{a}_{1}, \tilde{a}_{2}, \ldots, \tilde{a}_{n}\right)=\left(\bigoplus_{i_{1}, i_{2}, \ldots, i_{n}=1}^{n}\left(\hat{\otimes}_{j=1}^{n} \omega_{i_{j}} \tilde{a}_{i_{j}}^{* r_{j}}\right)\right)^{* 1 / \sum_{j=1}^{n} r_{j}},
$$

where $R=\left(r_{1}, r_{2}, \ldots, r_{n}\right)^{T}$ is the parameter vector with $r_{i} \geq 0(i=1,2, \ldots, n)$.

The DGNNNWBM operator can consider the relationship between any elements. Here are some special cases of it.

Remark 1. If $R=(\lambda, 0,0, \ldots, 0)^{T}$, that is, consider the relationship of a single element, then the DGNNNWBM reduces to:

$$
\operatorname{DGNNNWBM}_{\omega}^{R}\left(\tilde{a}_{1}, \tilde{a}_{2}, \ldots, \tilde{a}_{n}\right)=\left(\bigoplus_{i=1}^{n} \lambda \tilde{a}_{i}\right)^{* \frac{1}{\lambda}},
$$

which is called a generalized nonnegative normal neutrosophic weighted averaging (GNNNWA) operator. 
If $R=(s, t, 0,0, \ldots, 0)^{T}$, that is, consider the relationship between any two elements, then the DGNNNWBM reduces to:

$$
\operatorname{DGNNNWBM} M_{\omega}^{R}\left(\tilde{a}_{1}, \tilde{a}_{2}, \ldots, \tilde{a}_{n}\right)=\left(\bigoplus_{i, j=1}^{n}\left(\omega_{i} \tilde{a}_{i}^{* s} \hat{\otimes} \omega_{j} \tilde{a}_{j}^{* t}\right)\right)^{* \frac{1}{s+t}},
$$

which is the nonnegative normal neutrosophic weighted Bonferroni mean (NNNWBM) operator.

If $R=(s, t, r, 0,0, \ldots, 0)^{T}$, that is, consider the relationship between any three elements, then the DGNNNWBM reduces to:

$$
\text { DGNNNWBM } M_{\omega}^{R}\left(\tilde{a}_{1}, \tilde{a}_{2}, \ldots, \tilde{a}_{n}\right)=\left(\bigoplus_{i, j, k=1}^{n}\left(\omega_{i} \tilde{a}_{i}^{* s} \hat{\otimes} \omega_{j} \tilde{a}_{j}^{* t} \hat{\otimes} \omega_{k} \tilde{a}_{k}^{* r}\right)\right)^{* \frac{1}{s+t+r}},
$$

which is called a generalized nonnegative normal neutrosophic weighted Bonferroni mean (GNNNWBM) operator.

Theorem 2. Let $\left\{\tilde{a}_{i} \mid \tilde{a}_{i}=\left\langle\left(a_{i}, \sigma_{i}\right),\left(T_{i}, I_{i}, F_{i}\right)\right\rangle, i=1,2, \ldots, n\right\}$ be a set of NNNNs, then the aggregated result of the DGNNNWBM is also an NNNN and

where

$$
\operatorname{DGNNNWBM} M_{\omega}^{R}\left(\tilde{a}_{1}, \tilde{a}_{2}, \ldots, \tilde{a}_{n}\right)=\langle(a, \sigma),(T, I, F)\rangle,
$$

$$
\begin{gathered}
a=\left(\sum_{i_{1}, i_{2}, \ldots, i_{n}=1}^{n}\left(\prod_{j=1}^{n} \omega_{i_{j}} a_{i_{j}}^{r_{j}}\right)\right)^{\frac{1}{\sum_{j=1}^{n} r_{j}}}, \\
\sigma=\left(\sum_{i_{1}, i_{2}, \ldots, i_{n}=1}^{n}\left(\prod_{j=1}^{n} \omega_{i_{j}} \sigma_{i_{j}}^{r_{j}}\right)\right)^{\frac{1}{\sum_{j=1}^{n} r_{j}}}, \\
T=\left(1-\prod_{i_{1}, i_{2}, \ldots, i_{n}=1}^{n}\left(1-\prod_{j=1}^{n}\left(1-\left(1-T_{i_{j}}^{r_{j}}\right)^{\omega_{i_{j}}}\right)\right)\right)^{\frac{1}{\sum_{j=1}^{n} r_{j}}}, \\
I=1-\left(1-\prod_{i_{1}, i_{2}, \ldots, i_{n}=1}^{n}\left(1-\prod_{j=1}^{n}\left(1-\left(1-\left(1-I_{i_{j}}\right)^{r_{j}}\right)^{\omega_{i_{j}}}\right)\right)\right)^{\frac{1}{\sum_{j=1}^{n} r_{j}}}, \\
F=1-\left(1-\prod_{i_{1}, i_{2}, \ldots, i_{n}=1}^{n}\left(1-\prod_{j=1}^{n}\left(1-\left(1-\left(1-F_{i_{j}}\right)^{r_{j}}\right)^{\omega_{i_{j}}}\right)\right)\right)^{\frac{1}{\sum_{j=1}^{n} r_{j}}} .
\end{gathered}
$$

Proof. By Definition 5 and 12, we have

and

$$
\tilde{a}_{i_{j}}^{* r_{j}}=\left\langle\left(a_{i_{j}}^{r_{j}}, \sigma_{i_{j}}^{r_{j}}\right),\left(T_{i_{j}}^{r_{j}}, 1-\left(1-I_{i_{j}}\right)^{r_{j}}, 1-\left(1-F_{i_{j}}\right)^{r_{j}}\right)\right\rangle,
$$

$\omega_{i_{j}} \tilde{a}_{i_{j}}^{* r_{j}}=\left\langle\left(\omega_{i_{j}} a_{i_{j}}^{r_{j}}, \omega_{i_{j}} \sigma_{i_{j}}^{r_{j}}\right),\left(1-\left(1-T_{i_{j}}^{r_{j}}\right)^{\omega_{i_{j}}},\left(1-\left(1-I_{i_{j}}\right)^{r_{j}}\right)^{\omega_{i_{j}}},\left(1-\left(1-F_{i_{j}}\right)^{r_{j}}\right)^{\omega_{i_{j}}}\right)\right\rangle$,

so

$\hat{\otimes}_{j=1}^{n} \omega_{i_{j}} \tilde{a}_{i_{j}}^{* r^{j}}=$

$\left\langle\left(\prod_{j=1}^{n} \omega_{i_{j}} a_{i_{j}}^{r_{j}}, \prod_{j=1}^{n} \omega_{i_{j}} \sigma_{i_{j}}^{r_{j}}\right),\left(\prod_{j=1}^{n}\left(1-\left(1-T_{i_{j}}^{r_{j}}\right)^{\omega_{i_{j}}}\right)\right.\right.$,

$\left.\left.\sum_{j=1}^{n}\left(1-\left(1-I_{i_{j}}\right)^{r_{j}}\right)^{\omega_{i_{j}}}-\prod_{j=1}^{n}\left(1-\left(1-I_{i_{j}}\right)^{r_{j}}\right)^{\omega_{i_{j}}}, \sum_{j=1}^{n}\left(1-\left(1-F_{i_{j}}\right)^{r_{j}}\right)^{\omega_{i_{j}}}-\prod_{j=1}^{n}\left(1-\left(1-F_{i_{j}}\right)^{r_{j}}\right)^{\omega_{i_{j}}}\right)\right\rangle$

$=\left\langle\left(\prod_{j=1}^{n} \omega_{i_{j}} a_{i_{j}}^{r_{j}}, \prod_{j=1}^{n} \omega_{i_{j}} \sigma_{i_{j}}^{r_{j}}\right),\left(\prod_{j=1}^{n}\left(1-\left(1-T_{i_{j}}^{r_{j}}\right)^{\omega_{i_{j}}}\right)\right.\right.$,

$\left.\left.1-\prod_{j=1}^{n}\left(1-\left(1-\left(1-I_{i_{j}}\right)^{r_{j}}\right)^{\omega_{i_{j}}}\right), 1-\prod_{j=1}^{n}\left(1-\left(1-\left(1-F_{i_{j}}\right)^{r_{j}}\right)^{\omega_{i_{j}}}\right)\right)\right\rangle$,

then

$\bigoplus_{i_{1}, i_{2}, \ldots, i_{n}=1}^{n}\left(\hat{\otimes}_{j=1}^{n} \omega_{i_{j}} \tilde{a}_{i_{j}}^{* r_{j}}\right)$

$=\left\langle\left(\sum_{i_{1}, i_{2}, \ldots, i_{n}=1}^{n}\left(\prod_{j=1}^{n} \omega_{i_{j}} a_{i_{j}}^{r_{j}}\right), \sum_{i_{1}, i_{2}, \ldots, i_{n}=1}^{n}\left(\prod_{j=1}^{n} \omega_{i_{j}} \sigma_{i_{j}}^{r_{j}}\right)\right)\right.$,

$\left(i_{i_{1}, i_{2}, \ldots, i_{n}=1}^{n} \prod_{j=1}^{n}\left(1-\left(1-T_{i_{j}}^{r_{j}}\right)^{\omega_{i_{j}}}\right)-\prod_{i_{1}, i_{2}, \ldots, i_{n}=1}^{n} \prod_{j=1}^{n}\left(1-\left(1-T_{i_{j}}^{r_{j}}\right)^{\omega_{i_{j}}}\right)\right.$, 
$\left.\left.\prod_{i_{1}, i_{2}, \ldots i_{n}=1}^{n}\left(1-\prod_{j=1}^{n}\left(1-\left(1-\left(1-I_{i_{j}}\right)^{r_{j}}\right)^{\omega_{i_{j}}}\right)\right), \prod_{i_{1}, i_{2}, \ldots i_{n}=1}^{n}\left(1-\prod_{j=1}^{n}\left(1-\left(1-\left(1-F_{i_{j}}\right)^{r_{j}}\right)^{\omega_{i_{j}}}\right)\right)\right)\right\rangle$,

$=\left\langle\left(\sum_{i_{1}, i_{2}, \ldots, i_{n}=1}^{n}\left(\prod_{j=1}^{n} \omega_{i_{j}} a_{i_{j}}^{r_{j}}\right), \sum_{i_{1}, i_{2}, \ldots, i_{n}=1}^{n}\left(\prod_{j=1}^{n} \omega_{i_{j}} \sigma_{i_{j}}^{r_{j}}\right)\right),\left(1-\prod_{i_{1}, i_{2}, \ldots, i_{n}=1}^{n}\left(1-\prod_{j=1}^{n}\left(1-\left(1-T_{i_{j}}^{r_{j}}\right)^{\omega_{i_{j}}}\right)\right)\right.\right.$,

$\left.\left.\prod_{i_{1}, i_{2}, \ldots i_{n}=1}^{n}\left(1-\prod_{j=1}^{n}\left(1-\left(1-\left(1-I_{i_{j}}\right)^{r_{j}}\right)^{\omega_{i_{j}}}\right)\right), \prod_{i_{1}, i_{2}, \ldots i_{n}=1}^{n}\left(1-\prod_{j=1}^{n}\left(1-\left(1-\left(1-F_{i_{j}}\right)^{r_{j}}\right)^{\omega_{i_{j}}}\right)\right)\right)\right\rangle$,

Let

thus

Thereafter

$$
\begin{gathered}
a=\left(\sum_{i_{1}, i_{2}, \ldots, i_{n}=1}^{n}\left(\prod_{j=1}^{n} \omega_{i_{j}} a_{i_{j}}^{r_{j}}\right)\right)^{\frac{1}{\sum_{j=1}^{n} r_{j}}}, \\
\sigma=\left(\sum_{i_{1}, i_{2}, \ldots, i_{n}=1}^{n}\left(\prod_{j=1}^{n} \omega_{i_{j}} \sigma_{i_{j}}^{r_{j}}\right)\right)^{\frac{1}{\sum_{j=1}^{n} r_{j}}}, \\
T=\left(1-\prod_{i_{1}, i_{2}, \ldots, i_{n}=1}^{n}\left(1-\prod_{j=1}^{n}\left(1-\left(1-T_{i_{j}}^{r_{j}}\right)^{\omega_{i_{j}}}\right)\right)\right)^{\sum_{j=1}^{n} r_{j}}, \\
I=1-\left(1-\prod_{i_{1}, i_{2}, \ldots, i_{n}=1}^{n}\left(1-\prod_{j=1}^{n}\left(1-\left(1-\left(1-I_{i_{j}}\right)^{r_{j}}\right)^{\omega_{i_{j}}}\right)\right)\right)^{\frac{1}{\sum_{j=1}^{n} r_{j}}}, \\
F=1-\left(1-\prod_{i_{1}, i_{2}, \ldots, i_{n}=1}^{n}\left(1-\prod_{j=1}^{n}\left(1-\left(1-\left(1-F_{i_{j}}\right)^{r_{j}}\right)^{\omega_{i_{j}}}\right)\right)\right)^{\frac{1}{\sum_{j=1}^{n} r_{j}}} .
\end{gathered}
$$

$$
\left({\stackrel{\bigoplus}{i_{1}, i_{2}, \ldots, i_{n}=1}}_{n}^{n}\left(\hat{\otimes}_{j=1}^{n} \omega_{i_{j}} a_{i_{j}}^{* r_{j}}\right)\right)^{* 1 / \sum_{j=1}^{n} r_{j}}=\langle(a, \sigma),(T, I, F)\rangle .
$$

Hence

$$
a \geq 0, \sigma>0,0 \leq T \leq 1,0 \leq I \leq 1,0 \leq F \leq 1
$$

which completes the proof.

$$
0 \leq T+I+F \leq 3
$$

The following example is used to explain the calculation of the DGNNNWBM operator.

Example 2. Let $\tilde{a}_{1}=\langle(0.7,0.01),(0.6,0.2,0.1)\rangle, \tilde{a}_{2}=\langle(0.4,0.02),(0.8,0.1,0.3)\rangle$ be two NNNNs. With the weighted vector $\omega=(0.7,0.3)^{T}$, and the parameter vector $R=(2,3)^{T}$, then, according to Theorem 2 , we have $a=\left(\sum_{i_{i}, i_{2}=1}^{2}\left(\omega_{i_{1}} \omega_{i_{2}}\right)\right)^{\frac{1}{2+3}}$

$=\left(\omega_{1} a_{1}^{r_{1} \omega_{1} a_{1}^{r_{2}}}+\omega_{1} a_{1}^{r_{1} \omega_{2} a_{2}^{r_{2}}}+\omega_{2} a_{2}^{r_{1} \omega_{1} a_{1}^{r_{2}}}+\omega_{2} a_{2}^{r_{1} \omega_{2} a_{2}^{r_{2}}}\right)^{\frac{1}{5}}$

$=\left(0.7 \times 0.7^{2} \times 0.7 \times 0.7^{3}+0.7 \times 0.7^{2} \times 0.3 \times 0.4^{3}+0.3 \times 0.4^{2} \times 0.7 \times 0.7^{3}+0.3 \times 0.4^{2} \times 0.3 \times 0.4^{3}\right)^{\frac{1}{5}}$ $=0.6327$

Similarly, we can obtain $\sigma=0.0143$.

$T=\left(1-\prod_{i_{i}, i_{2}=1}^{2}\left(1-\left(1-\left(1-T_{i_{1}}^{r_{1}}\right)^{\omega_{i_{1}}}\right)\left(1-\left(1-T_{i_{2}}^{r_{2}}\right)^{\omega_{i_{2}}}\right)\right)\right)^{\frac{1}{2+3}}$

$=\left(1-\left(1-\left(1-\left(1-T_{1}^{r_{1}}\right)^{\omega_{1}}\right)\left(1-\left(1-T_{1}^{r_{2}}\right)^{\omega_{1}}\right)\right)\left(1-\left(1-\left(1-T_{1}^{r_{1}}\right)^{\omega_{1}}\right)\left(1-\left(1-T_{2}^{r_{2}}\right)^{\omega_{2}}\right)\right)(1-(1-(1-\right.$ $\left.\left.\left.\left.T_{2}^{r_{1}}\right)^{\omega_{2}}\right)\left(1-\left(1-T_{1}^{r_{2}}\right)^{\omega_{1}}\right)\right)\left(1-\left(1-\left(1-T_{2}^{r_{1}}\right)^{\omega_{2}}\right)\left(1-\left(1-T_{2}^{r_{2}}\right)^{\omega_{2}}\right)\right)\right)^{\frac{1}{5}}$

$=\left(1-\left(1-\left(1-\left(1-0.6^{2}\right)^{0.7}\right)\left(1-\left(1-0.6^{3}\right)^{0.7}\right)\right) \times\left(1-\left(1-\left(1-0.6^{2}\right)^{0.7}\right)\left(1-\left(1-0.8^{3}\right)^{0.3}\right)\right) \times(1-\right.$ $\left.\left.\left(1-\left(1-0.8^{2}\right)^{0.3}\right)\left(1-\left(1-0.6^{3}\right)^{0.7}\right)\right) \times\left(1-\left(1-\left(1-0.8^{2}\right)^{0.3}\right)\left(1-\left(1-0.8^{3}\right)^{0.4}\right)\right)\right)^{\frac{1}{5}}$

$=0.64$

$I=1-\left(1-\prod_{i_{i}, i_{2}=1}^{2}\left(1-\left(1-\left(1-\left(1-I_{i_{1}}\right)^{r_{1}}\right)^{\omega_{i_{1}}}\right)\left(1-\left(1-\left(1-I_{i_{2}}\right)^{r_{2}}\right)^{\omega_{i_{2}}}\right)\right)\right)^{\frac{1}{2+3}}$

$=1-\left(1-\left(1-\left(1-\left(1-\left(1-I_{1}\right)^{r_{1}}\right)^{\omega_{1}}\right)\left(1-\left(1-\left(1-I_{1}\right)^{r_{2}}\right)^{\omega_{1}}\right)\right)\left(1-\left(1-\left(1-\left(1-I_{1}\right)^{r_{1}}\right)^{\omega_{1}}\right)(1-(1-\right.\right.$ $\left.\left.\left.\left(1-I_{2}\right)^{r_{2}}\right)^{\omega_{2}}\right)\right)\left(1-\left(1-\left(1-\left(1-I_{2}\right)^{r_{1}}\right)^{\omega_{2}}\right)\left(1-\left(1-\left(1-I_{1}\right)^{r_{2}}\right)^{\omega_{1}}\right)\right)\left(1-\left(1-\left(1-\left(1-I_{2}\right)^{r_{1}}\right)^{\omega_{2}}\right)(1-\right.$ $\left.\left.\left.\left(1-\left(1-I_{2}\right)^{r_{2}}\right)^{\omega_{2}}\right)\right)\right)^{\frac{1}{5}}$ 
$=1-\left(1-\left(1-\left(1-\left(1-(1-0.2)^{2}\right)^{0.7}\right)\left(1-\left(1-(1-0.2)^{3}\right)^{0.7}\right)\right)\left(1-\left(1-\left(1-(1-0.2)^{2}\right)^{0.7}\right)(1-(1-\right.\right.$ $\left.\left.\left.(1-0.1)^{3}\right)^{0.3}\right)\right)\left(1-\left(1-\left(1-(1-0.1)^{2}\right)^{0.3}\right)\left(1-\left(1-(1-0.2)^{3}\right)^{0.7}\right)\right)\left(1-\left(1-\left(1-(1-0.1)^{2}\right)^{0.3}\right)(1-\right.$ $\left.\left.\left.\left(1-(1-0.1)^{3}\right)^{0.3}\right)\right)\right)^{\frac{1}{5}}$

$=0.1265$

Similarly, we can obtain $F=0.1195$.

So, DGNNNWBM $M_{\omega}^{R}\left(\tilde{a}_{1}, \tilde{a}_{2}\right)=\langle(0.6327,0.0143),(0.64,0.1265,0.1195)\rangle$

Next, we discuss some properties of the DGNNNWBM operator.

Theorem 3. (Monotonicity) Let $\left\{\tilde{a}_{i} \mid \tilde{a}_{i}=\left\langle\left(a_{i}, \sigma_{i}\right),\left(T_{i}, I_{i}, F_{i}\right)\right\rangle, i=1,2, \ldots, n\right\}$ and $\left\{\tilde{b}_{i} \mid \tilde{b}_{i}=\left\langle\left(b_{i}, \delta_{i}\right)\right.\right.$, $\left.\left.\left(T_{b_{i}}, I_{b_{i}}, F_{b_{i}}\right)\right\rangle, i=1,2, \ldots, n\right\}$ be two sets of NNNNs. If $a_{i} \leq b_{i}, \sigma_{i} \geq \delta_{i}$ and $T_{a_{i}}<T_{b_{i}}$ and $I_{a_{i}}>I_{b_{i}}$ and $F_{a_{i}}>F_{b_{i}}$ hold for all $i$, then

$$
\operatorname{DGNNNWBM} M_{\omega}^{R}\left(\tilde{a}_{1}, \tilde{a}_{2}, \ldots, \tilde{a}_{n}\right)<\operatorname{DGNNNWBM} M_{\omega}^{R}\left(\tilde{b}_{1}, \tilde{b}_{2}, \ldots, \tilde{b}_{n}\right),
$$

where $R=\left(r_{1}, r_{2}, \ldots, r_{n}\right)^{T}$ is the parameter vector with $r_{i} \geq 0(i=1,2, \ldots, n)$.

Proof. Let

$$
\begin{aligned}
& \text { DGNNNWBM } \\
& \text { DGNNNWBM } \\
& \operatorname{DGN}_{\omega}^{R}\left(\tilde{a}_{1}, \tilde{a}_{2}, \ldots, \tilde{b}_{2}, \ldots, \tilde{b}_{n}\right)=\left\langle(a, \sigma),\left(T_{a}, I_{a}, F_{a}\right)\right\rangle, \\
& =\left\langle(b, \delta),\left(T_{b}, I_{b}, F_{b}\right)\right\rangle .
\end{aligned}
$$

According to the DGNNNWBM operator, we have

$$
\begin{aligned}
& a=\left(\sum_{i_{1}, i_{2}, \ldots, i_{n}=1}^{n}\left(\prod_{j=1}^{n} \omega_{i_{j}} a_{i_{j}}^{r_{j}}\right)\right)^{1 / \sum_{j=1}^{n} r_{j}}, b=\left(\sum_{i_{1}, i_{2}, \ldots, i_{n}=1}^{n}\left(\prod_{j=1}^{n} \omega_{i_{j}} b_{i_{j}}^{r_{j}}\right)\right)^{1 / \sum_{j=1}^{n} r_{j}}, \\
& \sigma=\left(\sum_{i_{1}, i_{2}, \ldots, i_{n}=1}^{n}\left(\prod_{j=1}^{n} \omega_{i_{j}} \sigma_{i_{j}}^{r_{j}}\right)\right)^{1 / \sum_{j=1}^{n} r_{j}}, \delta=\left(\sum_{i_{1}, i_{2}, \ldots, i_{n}=1}^{n}\left(\prod_{j=1}^{n} \omega_{i_{j}} \delta_{i_{j}}^{r_{j}}\right)\right)^{1 / \sum_{j=1}^{n} r_{j}} .
\end{aligned}
$$

By $a_{i} \leq b_{i}, \sigma_{i} \geq \delta_{i}$ we get $a \leq b, \sigma \geq \delta$.

Let

when $T_{a_{i}}<T_{b_{i}}$, we can obtain

$$
\begin{aligned}
& T_{a}=\left(1-\prod_{i_{1}, i_{2}, \ldots, i_{n}=1}^{n}\left(1-\prod_{j=1}^{n}\left(1-\left(1-T_{a_{i_{j}}}^{r_{j}}\right)^{\omega_{i_{j}}}\right)\right)\right)^{\frac{1}{\sum_{j=1}^{n} r_{j}}}, \\
& T_{b}=\left(1-\prod_{i_{1}, i_{2}, \ldots, i_{n}=1}^{n}\left(1-\prod_{j=1}^{n}\left(1-\left(1-T_{b_{i_{j}}}^{r_{j}}\right)^{\omega_{i_{j}}}\right)\right)\right)^{\frac{1}{\sum_{j=1}^{n} r_{j}}}
\end{aligned}
$$

and

$$
\left(1-T_{a_{i_{j}}}^{r_{j}}\right)^{\omega_{i_{j}}}>\left(1-T_{b_{i_{j}}}^{r_{j}}\right)^{\omega_{i_{j}}}
$$

therefore

$$
1-\left(1-T_{a_{i_{j}}}^{r_{j}}\right)^{\omega_{i_{j}}}<1-\left(1-T_{b_{i_{j}}}^{r_{j}}\right)^{\omega_{i_{j}}},
$$

thus

$$
1-\prod_{j=1}^{n}\left(1-\left(1-T_{a_{i_{j}}}^{r_{j}}\right)^{\omega_{i_{j}}}\right)>1-\prod_{j=1}^{n}\left(1-\left(1-T_{b_{i_{j}}}^{r_{j}}\right)^{\omega_{i_{j}}}\right)
$$

$1-\prod_{i_{1}, i_{2}, \ldots, i_{n}=1}^{n}\left(1-\prod_{j=1}^{n}\left(1-\left(1-T_{a_{i_{j}}}^{r_{j}}\right)^{\omega_{i_{j}}}\right)\right)<1-\prod_{i_{1}, i_{2}, \ldots, i_{n}=1}^{n}\left(1-\prod_{j=1}^{n}\left(1-\left(1-T_{b_{i_{j}}}^{r_{j}}\right)^{\omega_{i_{j}}}\right)\right)$,

then $T_{a}<T_{b}$.

Similarly, we can obtain $I_{a}>I_{b}$ and $F_{a}>F_{b}$.

According to Definition 11,

$$
\begin{aligned}
& S\left(D G N N N W B M_{\omega}^{R}\left(\tilde{a}_{1}, \tilde{a}_{2}, \ldots, \tilde{a}_{n}\right)\right)=\left(a+\frac{1}{\sigma}\right)\left(2+T_{a}-I_{a}-F_{a}\right) \\
& <\left(b+\frac{1}{\delta}\right)\left(2+T_{b}-I_{b}-F_{b}\right)=S\left(D G N N N W B M_{\omega}^{R}\left(\tilde{b}_{1}, \tilde{b}_{2}, \ldots, \tilde{b}_{n}\right)\right) .
\end{aligned}
$$

Therefore, the proof is completed.

Remark 2. If $a_{i} \leq b_{i}, \sigma_{i} \geq \delta_{i}, T_{a_{i}} \leq T_{b_{i}}, I_{a_{i}} \geq I_{b_{i}}, F_{a_{i}} \geq F_{b_{i}}$ and $\left(T_{a_{i}}-T_{b_{i}}\right)^{2}+\left(I_{a_{i}}-I_{b_{i}}\right)^{2}+\left(F_{a_{i}}-F_{b_{i}}\right)^{2} \neq 0$ hold for any $i$, Theorem 3 is still holds. 
Theorem 4. (Boundedness) Let $\left\{\tilde{a}_{i} \mid \tilde{a}_{i}=\left\langle\left(a_{i}, \sigma_{i}\right),\left(T_{i}, I_{i}, F_{i}\right)\right\rangle, i=1,2, \ldots, n\right\}$ be a set of NNNNs. If $a^{+}=\left\langle\left(\max _{i}\left(a_{i}\right), \min _{i}\left(\sigma_{i}\right)\right),\left(\max _{i}\left(T_{i}\right), \min _{i}\left(I_{i}\right), \min _{i}\left(F_{i}\right)\right\rangle=\left\langle\left(a_{i}^{+}, \sigma_{i}^{+}\right),\left(T_{a_{i}}^{+}, I_{a_{i}}^{+}, F_{a_{i}}^{+}\right)\right\rangle\right.$and $a^{-}=$ $\left\langle\left(\min _{i}\left(a_{i}\right), \max _{i}\left(\sigma_{i}\right)\right),\left(\min _{i}\left(T_{i}\right), \max _{i}\left(I_{i}\right), \max _{i}\left(F_{i}\right)\right\rangle=\left\langle\left(a_{i}^{-}, \sigma_{i}^{-}\right),\left(T_{a_{i}}^{-}, I_{a_{i}}^{-}, F_{a_{i}}^{-}\right)\right\rangle\right.$, then

$\operatorname{DGNNNWBM}_{\omega}^{R}\left(\tilde{a}^{-}, \tilde{a}^{-}, \ldots, \tilde{a}^{-}\right) \leq \operatorname{DGNNNWBM}_{\omega}^{R}\left(\tilde{a}_{1}, \tilde{a}_{2}, \ldots, \tilde{a}_{n}\right) \leq \operatorname{DGNNNWBM}_{\omega}^{R}\left(\tilde{a}^{+}, \tilde{a}^{+}, \ldots, \tilde{a}^{+}\right)$.

Proof. By $a_{i}^{-} \leq a_{i} \leq a_{i}^{+}, \sigma_{i}^{+} \leq \sigma_{i} \leq \sigma_{i}^{-}, T_{i}^{-} \leq T_{i} \leq T_{i}^{+}, I_{i}^{+} \leq I_{i} \leq I_{i}^{-}, F_{i}^{+} \leq F_{i} \leq F_{i}^{-}$, according to Theorem 3 and Remark 2, we get

$\operatorname{DGNNNWBM} M_{\omega}^{R}\left(\tilde{a}^{-}, \tilde{a}^{-}, \ldots, \tilde{a}^{-}\right) \leq \operatorname{DGNNNWBM} M_{\omega}^{R}\left(\tilde{a}_{1}, \tilde{a}_{2}, \ldots, \tilde{a}_{n}\right) \leq \operatorname{DGNNNWBM}_{\omega}^{R}\left(\tilde{a}^{+}, \tilde{a}^{+}, \ldots, \tilde{a}^{+}\right)$.

Theorem 5. (Commutativity) Let $\left\{\tilde{a}_{i} \mid \tilde{a}_{i}=\left\langle\left(a_{i}, \sigma_{i}\right),\left(T_{i}, I_{i}, F_{i}\right)\right\rangle, i=1,2, \ldots, n\right\}$ be a set of NNNNs. If $\tilde{a}_{i}^{\prime}$ is any permutation of $\tilde{a}_{i}$, then

$$
\operatorname{DGNNNWBM} M_{\omega}^{R}\left(\tilde{a}_{1}, \tilde{a}_{2}, \ldots, \tilde{a}_{n}\right)=\operatorname{DGNNNWBM} M_{\omega}^{R}\left(\tilde{a}_{1}^{\prime}, \tilde{a}_{2}^{\prime}, \ldots, \tilde{a}_{n}^{\prime}\right) \text {. }
$$

Unfortunately, the DGNNNWBM operator is not satisfied with idempotency, i.e., $\operatorname{DGNNNWBM} M_{\omega}^{R}(\tilde{a}, \tilde{a}, \ldots, \tilde{a}) \neq a$.

Example 3. Let $\tilde{a}=\langle(4,0.2),(0.8,0.2,0.3)\rangle$ be an $N N N N$. The weighted vector $\omega=(0.25,0.25,0.25,0.25)^{T}$, and the parameter vector $R=(2,2,2,2)^{T}$, if all $\tilde{a}_{i}=\tilde{a}(i=1,2,3,4)$. Similar to Example 2, the following results can be obtained

$$
\operatorname{DGNNNWBM}_{\omega}^{R}(\tilde{a}, \tilde{a}, \tilde{a}, \tilde{a})=\langle(4,0.2),(0.9886,0.0114,0.0886)\rangle \neq \tilde{a} .
$$

Furthermore, we extend the DGWBGM to NNNNs and propose the dual generalized nonnegative normal neutrosophic weighted geometric Bonferroni mean (DGNNNWGBM) operator.

Definition 14. Suppose $\left\{\tilde{a}_{i} \mid \tilde{a}_{i}=\left\langle\left(a_{i}, \sigma_{i}\right),\left(T_{i}, I_{i}, F_{i}\right)\right\rangle, i=1,2, \ldots, n\right\}$ is a set of NNNNs with their weight vector being $\omega_{i}=\left(\omega_{1}, \omega_{2}, \ldots, \omega_{n}\right)^{T}$, where $\omega_{i} \in[0,1]$ and $\sum_{i=1}^{n} \omega_{i}=1$. The DGNNNWGBM operator is defined as

$$
\operatorname{DGNNNWGBM} M_{\omega}^{R}\left(\tilde{a}_{1}, \tilde{a}_{2}, \ldots, \tilde{a}_{n}\right)=\frac{1}{\sum_{j=1}^{n} r_{j}}\left(\hat{\otimes}_{i_{1}, i_{2}, \ldots i i_{n}=1}^{n}\left(\bigoplus_{j=1}^{n}\left(r_{j} \tilde{a}_{i_{j}}\right)\right)^{* \prod_{j=1}^{n} \omega_{i_{j}}}\right),
$$

where $R=\left(r_{1}, r_{2}, \ldots, r_{n}\right)^{T}$ is the parameter vector with $r_{i} \geq 0(i=1,2, \ldots, n)$.

The DGNNNWGBM operator can consider the relationship between any elements. Here are some special cases of it.

Remark 3. If $R=(\lambda, 0,0, \ldots, 0)^{T}$, that is, consider the relationship of a single element, then the DGNNNWGBM reduces to:

$$
\operatorname{DGNNNWGBM} M_{\omega}^{R}\left(\tilde{a}_{1}, \tilde{a}_{2}, \ldots, \tilde{a}_{n}\right)=\frac{1}{\lambda}\left(\hat{\otimes}_{i=1}^{n}\left(\lambda \tilde{a}_{i}\right)^{* \omega_{i}}\right)
$$

which is called a generalized nonnegative normal neutrosophic weighted geometric averaging (GNNNWGA) operator.

If $R=(s, t, 0,0, \ldots, 0)^{T}$, that is, consider the relationship between any two elements, then the DGNNNWGBM reduces to:

$$
\operatorname{DGNNNWGBM} M_{\omega}^{R}\left(\tilde{a}_{1}, \tilde{a}_{2}, \ldots, \tilde{a}_{n}\right)=\frac{1}{s+t} \hat{\bigotimes}_{i, j=1}^{n}\left(s \tilde{a}_{i} \oplus t \tilde{a}_{j}\right)^{* \omega_{i} \omega_{j}}
$$

which is called a nonnegative normal neutrosophic weighted Bonferroni geometric (NNNWBG) operator.

If $R=(s, t, r, 0,0, \ldots, 0)^{T}$, that is, consider the relationship between any three elements, then the DGNNNWGBM reduces to:

$$
\text { DGNNNWGBM } M_{\omega}^{R}\left(\tilde{a}_{1}, \tilde{a}_{2}, \ldots, \tilde{a}_{n}\right)=\frac{1}{s+t+r} \hat{\otimes}_{i, j, k=1}^{n}\left(s \tilde{a}_{i} \oplus t \tilde{a}_{j} \oplus t \tilde{a}_{k}\right)^{* \omega_{i} \omega_{j} \omega_{k}}
$$

which is called a generalized nonnegative normal neutrosophic weighted Bonferroni geometric (GNNNWBG) operator. 
Theorem 6. Let $\left\{\tilde{a}_{i} \mid \tilde{a}_{i}=\left\langle\left(a_{i}, \sigma_{i}\right),\left(T_{i}, I_{i}, F_{i}\right)\right\rangle, i=1,2, \ldots, n\right\}$ be a set of NNNNs, then the aggregated result of DGNNNWGBM is also an NNNN and

where

$$
\operatorname{DGNNNWGBM} M_{\omega}^{R}\left(\tilde{a}_{1}, \tilde{a}_{2}, \ldots, \tilde{a}_{n}\right)=\langle(\hat{a}, \hat{\sigma}),(\hat{T}, \hat{I}, \hat{F})\rangle,
$$

$$
\begin{gathered}
\hat{a}=\frac{1}{\sum_{j=1}^{n} r_{j}} \prod_{i_{1}, i_{2}, \ldots i_{n}=1}^{n}\left(\sum_{j=1}^{n}\left(r_{j} a_{i_{j}}\right)\right)^{\prod_{j=1}^{n} \omega_{i_{j}}}, \\
\hat{\sigma}=\frac{1}{\sum_{j=1}^{n} r_{j}} \prod_{i_{1}, i_{2}, \ldots i_{n}=1}^{n}\left(\sum_{j=1}^{n}\left(r_{j} \sigma_{i_{j}}\right)\right)^{\prod_{j=1}^{n} \omega_{i_{j}}}, \\
\hat{T}=1-\left(1-\prod_{i_{1}, i_{2}, \ldots, i_{n}=1}^{n}\left(1-\prod_{j=1}^{n}\left(1-T_{i_{j}}\right)^{r_{j}}\right)^{\prod_{j=1}^{n} \omega_{i_{j}}}\right)^{\frac{1}{\sum_{j=1}^{n} r_{j}}}, \\
\hat{I}=\left(1-\prod_{i_{1}, i_{2}, \ldots, i_{n}=1}^{n}\left(\left(1-\prod_{j=1}^{n} I_{i_{j}}^{r_{j}}\right)^{\prod_{j=1}^{n} \omega_{i_{j}}}\right)\right)^{\frac{1}{\sum_{j=1}^{n} r_{j}}}, \\
\hat{F}=\left(1-\prod_{i_{1}, i_{2}, \ldots, i_{n}=1}^{n}\left(\left(1-\prod_{j=1}^{n} F_{i_{j}}^{r_{j}}\right)^{\prod_{j=1}^{n} \omega_{i_{j}}}\right)\right)^{\frac{1}{\sum_{j=1}^{n} r_{j}}} .
\end{gathered}
$$

The proof of Theorem 6 is similar to that of Theorem 2.

Likewise, an example is used to explain the calculation of the DGNNNWGBM operator.

Example 4. Let $\tilde{a}_{1}=\langle(0.5,0.03),(0.5,0.4,0.1)\rangle, \tilde{a}_{2}=\langle(0.8,0.015),(0.9,0.2,0.2)\rangle$ be two NNNNs. The weighted vector $\omega=(0.6,0.4)^{T}$, and the parameter vector $R=(3,4)^{T}$, then, according to Theorem 6 , we have

$\hat{a}=\frac{1}{3+4} \prod_{i_{1}, i_{2}=1}^{2}\left(r_{1} a_{i_{1}}+r_{2} a_{i_{2}}\right)^{\omega_{i_{1}} \omega_{i_{2}}}$

$=\frac{1}{7}\left(r_{1} a_{1}+r_{2} a_{1}\right)^{\omega_{1} \omega_{1}}\left(r_{1} a_{1}+r_{2} a_{2}\right)^{\omega_{1} \omega_{2}}\left(r_{1} a_{2}+r_{2} a_{1}\right)^{\omega_{2} \omega_{1}}\left(r_{1} a_{2}+r_{2} a_{2}\right)^{\omega_{2} \omega_{2}}$

$=\frac{1}{7}(3 \times 0.5+4 \times 0.5)^{0.6 \times 0.6}(3 \times 0.5+4 \times 0.8)^{0.6 \times 0.4}(3 \times 0.8+4 \times 0.5)^{0.4 \times 0.6}(3 \times 0.8+4 \times 0.8)^{0.4 \times 0.4}$

$=0.6112$

Similarly, we can obtain $\hat{\sigma}=0.0234$.

$\hat{T}=1-\left(1-\prod_{i_{1}, i_{2}=1}^{2}\left(1-\left(1-T_{i_{1}}\right)^{r_{1}}\left(1-T_{i_{2}}\right)^{r_{2}}\right)^{\omega_{i_{1}} \omega_{i_{2}}}\right)^{\frac{1}{3+4}}$

$=1-\left(1-\left(1-\left(1-T_{1}\right)^{r_{1}}\left(1-T_{1}\right)^{r_{2}}\right)^{\omega_{1} \omega_{1}}\left(1-\left(1-T_{1}\right)^{r_{1}}\left(1-T_{2}\right)^{r_{2}}\right)^{\omega_{1} \omega_{2}}\left(1-\left(1-T_{2}\right)^{r_{1}}(1-\right.\right.$ $\left.\left.\left.T_{1}\right)^{r_{2}}\right)^{\omega_{2} \omega_{1}}\left(1-\left(1-T_{2}\right)^{r_{1}}\left(1-T_{2}\right)^{r_{2}}\right)^{\omega_{2} \omega_{2}}\right)^{\frac{1}{7}}$

$=1-\left(1-\left(1-(1-0.5)^{3}(1-0.5)^{4}\right)^{0.6 \times 0.6} \times\left(1-(1-0.5)^{3}(1-0.9)^{4}\right)^{0.6 \times 0.4} \times\left(1-(1-0.9)^{3}(1-\right.\right.$ $\left.\left.0.5)^{4}\right)^{0.4 \times 0.6}\left(1-(1-0.9)^{3}(1-0.9)^{4}\right)^{0.4 \times 0.4}\right)^{\frac{1}{7}}$

$=0.5674$

$\hat{I}=\left(1-\prod_{i_{1}, i_{2}=1}^{2}\left(1-I_{i_{1}}^{r_{1}} I_{i_{2}}^{r_{2}}\right)^{\omega_{i_{1}} \omega_{i_{2}}}\right)^{\frac{1}{3+4}}$

$=\left(1-\left(1-I_{1}^{r_{1}} I_{1}^{r_{2}}\right)^{\omega_{1} \omega_{1}}\left(1-I_{1}^{r_{1}} I_{2}^{r_{2}}\right)^{\omega_{1} \omega_{2}}\left(1-I_{2}^{r_{1}} I_{1}^{r_{2}}\right)^{\omega_{2} \omega_{1}}\left(1-I_{2}^{r_{1}} I_{2}^{r_{2}}\right)^{\omega_{2} \omega_{2}}\right)^{\frac{1}{7}}$

$=\left(1-\left(1-0.4^{3} \times 0.4^{4}\right)^{0.6 \times 0.6}\left(1-0.4^{3} \times 0.2^{4}\right)^{0.6 \times 0.4}\left(1-0.2^{3} \times 0.4^{4}\right)^{0.4 \times 0.6}\left(1-0.2^{3} \times 0.2^{4}\right)^{0.4 \times 0.4}\right)^{\frac{1}{7}}$

$=0.3517$

Similarly, we can obtain $\hat{I}=0.1598$.

So, DGNNNWGBM $M_{\omega}^{R}\left(\tilde{a}_{1}, \tilde{a}_{2}\right)=\langle(0.6112,0.0234),(0.5674,0.3517,0.1598)\rangle$.

The DGNNNWGBM operator has the same properties as the DGNNNWBM operator. The proof is also similar to that of the DGNNNWBM operator. Of particular note, the DGNNNWGBM operator satisfies the property of idempotency.

Theorem 7. (Idempotency) Let $\left\{\tilde{a}_{i} \mid \tilde{a}_{i}=\left\langle\left(a_{i}, \sigma_{i}\right),\left(T_{i}, I_{i}, F_{i}\right)\right\rangle, i=1,2, \ldots, n\right\}$ be a set of NNNNs. If all $\tilde{a}_{i}=\tilde{a}$, then

$$
\operatorname{DGNNNWGBM} M_{\omega}^{R}\left(\tilde{a}_{1}, \tilde{a}_{2}, \ldots, \tilde{a}_{n}\right)=\tilde{a} .
$$


Proof. Since $\tilde{a}_{i}=\tilde{a}(i=1,2, \ldots, n)$, according to operational rules,

$$
\begin{aligned}
& \text { DGNNNWGBM } M_{\omega}^{R}\left(\tilde{a}_{1}, \tilde{a}_{2}, \ldots, \tilde{a}_{n}\right) \\
& =\frac{1}{\sum_{j=1}^{n} r_{j}}\left(\hat{\bigotimes}_{i_{1}, i_{2}, \ldots i_{n}=1}^{n}\left(\bigoplus_{j=1}^{n}\left(r_{j} \tilde{a}_{i_{j}}\right)\right)^{* \prod_{j=1}^{n} \omega_{i_{j}}}\right) \\
& =\frac{1}{\sum_{j=1}^{n} r_{j}}\left(\hat{\bigotimes}_{i_{1}, i_{2}, \ldots, i_{n}=1}^{n}\left(\sum_{j=1}^{n} r_{j} \tilde{a}\right)^{* \prod_{j=1}^{n} \omega_{i_{j}}}\right) \\
& =\frac{1}{\sum_{j=1}^{n} r_{j}}\left(\sum_{j=1}^{n} r_{j} \tilde{a}\right)^{*}{ }_{i_{1}, i_{2}, \ldots, i_{n}=1}^{n} \prod_{j=1}^{n} \omega_{i_{j}}
\end{aligned}
$$

Here, $\sum_{i_{1}, i_{2}, \ldots, i_{n}=1}^{n} \prod_{j=1}^{m} \omega_{i_{j}}=1$ is proved by mathematical induction.

When $m=2$, we have $\sum_{i_{1}, i_{2}=1}^{n} \omega_{i_{1}} \omega_{i_{2}}=\sum_{i_{1}=1}^{n} \omega_{i_{1}} \sum_{i_{2}=1}^{n} \omega_{i_{2}}=1$.

Suppose $m=k-1$, and $\sum_{i_{1}, i_{2}, \ldots, i_{k-1}=1}^{n} \prod_{j=1}^{k-1} \omega_{i_{j}}=1$,

so when $m=k$, we get

Then

$$
\sum_{i_{1}, i_{2}, \ldots, i_{k}=1}^{n} \prod_{j=1}^{k} \omega_{i_{j}}=\sum_{i_{1}, i_{2}, \ldots, i_{k}=1}^{n} \prod_{j=1}^{k-1} \omega_{i_{j}} \omega_{i_{k}}=\sum_{i_{1}, i_{2}, \ldots, i_{k-1}=1}^{n} \prod_{j=1}^{k-1} \omega_{i_{j}} \sum_{i_{k}=1}^{k} \omega_{i_{k}}=1 .
$$

That completes the proof.

$$
\operatorname{DGNNNWGBM} M_{\omega}^{R}\left(\tilde{a}_{1}, \tilde{a}_{2}, \ldots, \tilde{a}_{n}\right)=\tilde{a} .
$$

Theorem 8. (Monotonicity) Let $\left\{\tilde{a}_{i} \mid \tilde{a}_{i}=\left\langle\left(a_{i}, \sigma_{i}\right),\left(T_{i}, I_{i}, F_{i}\right)\right\rangle, i=1,2, \ldots, n\right\}$ and $\left\{\tilde{b}_{i} \mid \tilde{b}_{i}=\left\langle\left(b_{i}, \delta_{i}\right)\right.\right.$, $\left.\left.\left(T_{b_{i}}, I_{b_{i}}, F_{b_{i}}\right)\right\rangle, i=1,2, \ldots, n\right\}$ be two sets of NNNNs. If $a_{i} \leq b_{i}, \sigma_{i} \geq \delta_{i}, T_{a_{i}} \leq T_{b_{i}}, I_{a_{i}} \geq I_{b_{i}}, F_{a_{i}} \geq F_{b_{i}}$ and $\left(T_{a_{i}}-T_{b_{i}}\right)^{2}+\left(I_{a_{i}}-I_{b_{i}}\right)^{2}+\left(F_{a_{i}}-F_{b_{i}}\right)^{2} \neq 0$ hold for any $i$, then

$$
\text { DGNNNWGBM } M_{\omega}^{R}\left(\tilde{a}_{1}, \tilde{a}_{2}, \ldots, \tilde{a}_{n}\right)<\operatorname{DGNNNWGBM} M_{\omega}^{R}\left(\tilde{b}_{1}, \tilde{b}_{2}, \ldots, \tilde{b}_{n}\right) \text {. }
$$

Theorem 9. (Boundedness) Let $\left\{\tilde{a}_{i} \mid \tilde{a}_{i}=\left\langle\left(a_{i}, \sigma_{i}\right),\left(T_{i}, I_{i}, F_{i}\right)\right\rangle, i=1,2, \ldots, n\right\}$ be a set of NNNNs. If $\tilde{a}^{+}=$ $\left\langle\left(\max _{i}\left(a_{i}\right), \min _{i}\left(\sigma_{i}\right)\right),\left(\max _{i}\left(T_{i}\right), \min _{i}\left(I_{i}\right), \min _{i}\left(F_{i}\right)\right\rangle\right.$ and $\tilde{a}^{-}=\left\langle\left(\min _{i}\left(a_{i}\right), \max _{i}\left(\sigma_{i}\right)\right),\left(\min _{i}\left(T_{i}\right), \max _{i}\left(I_{i}\right)\right.\right.$, $\left.\max _{i}\left(F_{i}\right)\right\rangle$, then

$$
\tilde{a}^{-} \leq \operatorname{DGNNNWGBM} M_{\omega}^{R}\left(\tilde{a}_{1}, \tilde{a}_{2}, \ldots, \tilde{a}_{n}\right) \leq \tilde{a}^{+}
$$

Theorem 10. (Commutativity) Let $\left\{\tilde{a}_{i} \mid \tilde{a}_{i}=\left\langle\left(a_{i}, \sigma_{i}\right),\left(T_{i}, I_{i}, F_{i}\right)\right\rangle, i=1,2, \ldots, n\right\}$ be a set of NNNNs. $\tilde{a}_{i}^{\prime}$ is any permutation of $\tilde{a}_{i}$, then

$$
\operatorname{DGNNNWGBM} M_{\omega}^{R}\left(\tilde{a}_{1}, \tilde{a}_{2}, \ldots, \tilde{a}_{n}\right)=\operatorname{DGNNNWGBM} M_{\omega}^{R}\left(\tilde{a}_{1}^{\prime}, \tilde{a}_{2}^{\prime}, \ldots, \tilde{a}_{n}^{\prime}\right) .
$$

\section{A Multiple Attribute Decision-Making Method on the Basis of the DGNNNWBM Operator and DGNNNWGBM Operator}

In this section, based on the NNNN, we utilize the DGNNNWBM operator or DGNNNWGBM operator to solve the MADM problem.

Let $A=\left\{A_{1}, A_{2}, \ldots, A_{m}\right\}$ be a set of the alternatives, and $C=\left\{C_{1}, C_{2}, \ldots, C_{n}\right\}$ be a set of the attributes; the weight vector of the attribute is $\omega=\left(\omega_{1}, \omega_{2}, \ldots, \omega_{n}\right)^{T}$, where $\omega_{j} \in[0,1]$ and $\sum_{j=1}^{n} \omega_{j}=1$. Let $D=\left(\tilde{a}_{i j}\right)_{m \times n}$ be the decision matrix, and $\tilde{a}_{i j}=\left\langle\left(a_{i j}, \sigma_{i j}\right),\left(T_{i j}, I_{i j}, F_{i j}\right)\right\rangle$ be the evaluation value of the alternative $A_{i}$ with respect to attribute $C_{j}$, denoted by the form of NNNN.

The DGNNNWBM operator or DGNNNWGBM operator can be used to handle the MADM problem, and the steps are shown as follows:

Step 1. Standardize the decision matrix. 
If all the attributes $C_{i}$ are of the same type, then the attribute values do not need standardization. If there is a different type, the attributes should be converted so they are of the same type. Suppose the decision matrix $D=\left(\tilde{a}_{i j}\right)_{m \times n}$ transforms to the standardized matrix $\tilde{D}=\left(\tilde{a}_{i j}^{\prime}\right)_{m \times n}$.

According to [6], we have the following standardization method. For the benefit attribute:

For the cost attribute:

$$
\tilde{a}_{i j}^{\prime}=\left\langle\left(\frac{a_{i j}}{\max _{1 \leq i \leq m}\left(a_{i j}\right)}, \frac{\sigma_{i j}}{\max _{1 \leq i \leq m}\left(\sigma_{i j}\right)} \frac{\sigma_{i j}}{a_{i j}}\right),\left(T_{i j}, I_{i j}, F_{i j}\right)\right\rangle \text {. }
$$

$$
\tilde{a}_{i j}^{\prime}=\left\langle\left(\frac{\min _{1 \leq i \leq m}\left(a_{i j}\right)}{a_{i j}}, \frac{\sigma_{i j}}{\max _{1 \leq i \leq m}\left(\sigma_{i j}\right)} \frac{\sigma_{i j}}{a_{i j}}\right),\left(F_{i j}, 1-I_{i j}, T_{i j}\right)\right\rangle .
$$

Step 2. Utilize the DGNNNWBM operator

or the DGNNNWGBM operator

$$
\tilde{a}_{i}=\operatorname{DGNNNWBM} M_{\omega}^{R}\left(\tilde{a}_{i 1}, \tilde{a}_{i 2}, \ldots, \tilde{a}_{i n}\right)=\left\langle\left(a_{i}, \sigma_{i}\right),\left(T_{i}, I_{i}, F_{i}\right)\right\rangle
$$

for comprehensive evaluation.

$$
\tilde{a}_{i}=\operatorname{DGNNNWGBM} M_{\omega}^{R}\left(\tilde{a}_{i 1}, \tilde{a}_{i 2}, \ldots, \tilde{a}_{i n}\right)=\left\langle\left(a_{i}, \sigma_{i}\right),\left(T_{i}, I_{i}, F_{i}\right)\right\rangle
$$

Step 3. According to rank principles, which are shown in Definitions 10 and 11, rank the alternatives $A_{1}, A_{2}, \ldots, A_{m}$ and choose the best one.

\section{Numerical Example and Comparative Analysis}

In this section, the effectiveness of the proposed MADM method is illustrated, demonstrating the effect of different parameter values on the final ranking results. Finally, the advantages of the proposed method are illustrated by comparison.

\subsection{The Numerical Example}

In the following, the application of the proposed method is illustrated by a numerical example.

Example 5. Patients choose a hospital according to their own needs. There are five alternatives hospitals to choose from: (1) $A_{1}$ is a people's hospital; (2) $A_{2}$ is a city hospital; (3) $A_{3}$ is a second city hospital; (4) $A_{4}$ is the first affiliated hospital; and (5) $A_{5}$ is the second affiliated hospital. There are four evaluation attributes: (1) $C_{1}$ is the hardware and software facilities; (2) $C_{2}$ is the physician team; (3) $C_{3}$ is the consumption index; and (4) $C_{4}$ is the service quality. We know the attributes $C_{1}, C_{2}$, and $C_{4}$ are benefit criteria, and $C_{3}$ is cost. The weight vector of the attributes is $\omega=(0.2,0.4,0.3,0.1)^{T}$. The final evaluation outcomes are expressed by the NNNN, which is shown in Table 3.

Table 3. The nonnegative normal neutrosophic decision matrix $D$.

\begin{tabular}{ccccc}
\hline & $C_{1}$ & $C_{2}$ & $C_{3}$ & $C_{4}$ \\
\hline$A_{1}$ & $\langle(4,0.3),(0.7,0.2,0.3)\rangle$ & $\langle(7,0.7),(0.6,0.1,0.1)\rangle$ & $\langle(5.5,0.6),(0.3,0.3,0.6)\rangle$ & $\langle(6,0.4),(0.7,0.2,0.4)\rangle$ \\
$A_{2}$ & $\langle(5,0.2),(0.5,0.4,0.5)\rangle$ & $\langle(8,0.5),(0.7,0.2,0.3)\rangle$ & $\langle(6,0.2),(0.2,0.1,0.7)\rangle$ & $\langle(7,0.6),(0.4,0.2,0.7)\rangle$ \\
$A_{3}$ & $\langle(3,0.5),(0.3,0.3,0.4)\rangle$ & $\langle(6,0.2),(0.4,0.5,0.3)\rangle$ & $\langle(4,0.7),(0.3,0.5,0.5)\rangle$ & $\langle(5.5,0.4),(0.5,0.4,0.2)\rangle$ \\
$A_{4}$ & $\langle(4.5,0.6),(0.3,0.5,0.3)\rangle$ & $\langle(5,0.4),(0.6,0.4,0.5)\rangle$ & $\langle(7,0.4),(0.3,0.3,0.5)\rangle$ & $\langle(4,0.5),(0.8,0.2,0.5)\rangle$ \\
$A_{5}$ & $\langle(6,0.5),(0.8,0.1,0.2)\rangle$ & $\langle(6.5,0.6),(0.8,0.2,0.4)\rangle$ & $\langle(5,0.3),(0.4,0.2,0.6)\rangle$ & $\langle(5,0.6),(0.5,0.5,0.2)\rangle$ \\
\hline
\end{tabular}

Step 1. Since $C_{1}, C_{2}$, and $C_{4}$ are benefit attributes, we have

$a_{11}^{\prime}=\frac{a_{11}}{\max _{1 \leq i \leq 5} a_{i 1}}=\frac{4}{6}=0.6667, \sigma_{11}^{\prime}=\frac{\sigma_{11}}{\max _{1 \leq i \leq 5} \sigma_{i 1}} \frac{\sigma_{11}}{a_{11}}=\frac{0.3}{0.6} \frac{0.3}{4}=0.0375, T_{11}^{\prime}=T_{11}, I_{11}^{\prime}=I_{11}, I_{11}^{\prime}=I_{11}$, and $C_{3}$ is the cost attribute, so we have

$$
\begin{gathered}
a_{13}^{\prime}=\frac{m i_{1 \leq i \leq 5}}{a_{13}}=\frac{4}{5.5}=0.7273, \sigma_{13}=\frac{\sigma_{13}}{m a x_{1 \leq i \leq 5} \sigma_{11}} \frac{\sigma_{13}}{a_{13}}=\frac{0.6}{0.7} \frac{0.6}{5.5}=0.0935, \\
T_{13}^{\prime}=F_{13}=0.6, I_{13}^{\prime}=1-I_{13}=0.7, F_{13}^{\prime}=T_{13}=0.3 .
\end{gathered}
$$

The normalized decision matrix is shown in Table 4. 
Table 4. Normalized decision matrix $\tilde{D}$.

\begin{tabular}{ccc}
\hline & $\boldsymbol{C}_{\mathbf{1}}$ & $\boldsymbol{C}_{\mathbf{2}}$ \\
\hline$A_{1}$ & $\langle(0.6667,0.0375),(0.7,0.2,0.3)\rangle$ & $\langle(0.85,0.1),(0.6,0.1,0.1)\rangle$ \\
$A_{2}$ & $\langle(0.8333,0.0133),(0.5,0.4,0.5)\rangle$ & $\langle(1,0.0446),(0.7,0.2,0.3)\rangle$ \\
$A_{3}$ & $\langle(0.5,0.1389),(0.3,0.3,0.4)\rangle$ & $\langle(0.75,0.0095),(0.4,0.5,0.3)\rangle$ \\
$A_{4}$ & $\langle(0.75,0.1333),(0.3,0.5,0.3)\rangle$ & $\langle(0.625,0.0457),(0.6,0.4,0.5)\rangle$ \\
$A_{5}$ & $\langle(1,0.0694),(0.8,0.1,0.2)\rangle$ & $\langle(0.8125,0.0791),(0.8,0.2,0.4)\rangle$ \\
\hline & $C_{\mathbf{3}}$ & $\boldsymbol{C}_{\mathbf{4}}$ \\
\hline$A_{1}$ & $\langle(0.7273,0.0935),(0.6,0.7,0.3)\rangle$ & $\langle(0.8571,0.0444),(0.7,0.2,0.4)\rangle$ \\
$A_{2}$ & $\langle(0.6667,0.0095),(0.7,0.9,0.2)\rangle$ & $\langle(1,0.0857),(0.4,0.2,0.7)\rangle$ \\
$A_{3}$ & $\langle(1,0.1750),(0.5,0.5,0.3)\rangle$ & $\langle(0.7857,0.0485),(0.5,0.4,0.2)\rangle$ \\
$A_{4}$ & $\langle(0.5714,0.0327),(0.5,0.7,0.3)\rangle$ & $\langle(0.5714,0.1042),(0.8,0.2,0.5)\rangle$ \\
$A_{5}$ & $\langle(0.8,0.0257),(0.6,0.8,0.4)\rangle$ & $\langle(0.7143,0.12),(0.5,0.5,0.2)\rangle$ \\
\hline
\end{tabular}

Step 2. Calculate the comprehensive evaluation value of each alternative by using the DGNNNWBM (DGNNNWGBM) operator (suppose $R=(1,1,1,1)^{T}$ ), which is shown in Table 5. (There are 256 cases in this example, which are not listed here. MATLAB can be used for the calculations.)

Table 5. Utilization of the dual generalized nonnegative normal neutrosophic weighted Bonferroni mean (DGNNNWBM) operator and dual generalized nonnegative normal neutrosophic weighted geometric Bonferroni mean (DGNNNWGBM) operator $\mathrm{R}=(1,1,1,1)$.

\begin{tabular}{ccc}
\hline & DGNNNWBM & DGNNNWGBM \\
\hline$A_{1}$ & $\langle(0.7772,0.08),(0.8161,0.0525,0,0303)\rangle$ & $\langle(0.7763,0.0788),(0.6299,0.313,0.2301)\rangle$ \\
$A_{2}$ & $\langle(0.8667,0.0319),(0.8095,0.2210,0.1474)\rangle$ & $\langle(0.8636,0.0295),(0.6296,0.4622,0.3507)\rangle$ \\
$A_{3}$ & $\langle(0.7786,0.0889),(0.5042,0.2935,0.1071)\rangle$ & $\langle(0.7736,0.0788),(0.4194,0.4503,0.31)\rangle$ \\
$A_{4}$ & $\langle(0.6286,0.0652),(0.6902,0.3319,0.2185)\rangle$ & $\langle(0.6277,0.0622),(0.5287,0.4919,0.4004)\rangle$ \\
$A_{5}$ & $\langle(0.8364,0.0652),(0.9198,0.1201,0.1236)\rangle$ & $\langle(0.8353,0.0635),(0.7098,0.3965,0.3402)\rangle$ \\
\hline
\end{tabular}

Step 3. According to Definition 10, for the DGNNNWBM operator,

$$
\begin{gathered}
S\left(A_{1}\right)=\left(0.7772+\frac{1}{0.08}\right)(2+0.8161-0.0525-0.0303), \\
S\left(A_{2}\right)=78.59, S\left(A_{3}\right)=25.29, S\left(A_{4}\right)=34.18, S\left(A_{5}\right)=43.26 .
\end{gathered}
$$

By the ranking principle of Definition 11, we obtain $A_{2}>A_{5}>A_{1}>A_{4}>A_{3}$, which is shown in Table 6. The best alternative is $A_{2}$.

Table 6. The score of the alternatives.

\begin{tabular}{ccccccc}
\hline & $S\left(A_{\mathbf{1}}\right)$ & $S\left(A_{\mathbf{2}}\right)$ & $S\left(A_{\mathbf{3}}\right)$ & $S\left(A_{\mathbf{4}}\right)$ & $S\left(A_{\mathbf{5}}\right)$ & Ranking \\
\hline DGNNNWBM & 36.29 & 78.59 & 25.29 & 34.18 & 43.26 & $A_{2}>A_{5}>A_{1}>A_{4}>A_{3}$ \\
DGNNNWGBM & 28.10 & 63.07 & 22.34 & 27.33 & 32.73 & $A_{2}>A_{5}>A_{4}>A_{1}>A_{3}$ \\
\hline
\end{tabular}

\subsection{Influence Analysis}

To show the effects on the ranking results by altering the parameters of the DGNNNWBM and DGNNNWGBM operators, according to Definition 10 and 11, we can get the results by using MATLAB, which is shown in Tables 7 and 8. 
Table 7. Ranking for different parameters of DGNNNWBM.

\begin{tabular}{ccccccc}
\hline $\boldsymbol{R}$ & $S\left(\boldsymbol{A}_{\mathbf{1}}\right)$ & $\boldsymbol{S}\left(\boldsymbol{A}_{\mathbf{2}}\right)$ & $\boldsymbol{S}\left(\boldsymbol{A}_{\mathbf{3}}\right)$ & $\boldsymbol{S}\left(\boldsymbol{A}_{\mathbf{4}}\right)$ & $\boldsymbol{S}\left(\boldsymbol{A}_{\mathbf{5}}\right)$ & Ranking \\
\hline$(1,1,1,1)$ & 36.29 & 78.59 & 25.29 & 34.18 & 43.26 & $A_{2}>A_{5}>A_{1}>A_{4}>A_{3}$ \\
$(3,3,3,3)$ & 28.85 & 47.48 & 14.96 & 22.25 & 31.82 & $A_{2}>A_{5}>A_{1}>A_{4}>A_{3}$ \\
$(4,4,4,4)$ & 27.84 & 42.48 & 14.06 & 20.61 & 30.08 & $A_{2}>A_{5}>A_{1}>A_{4}>A_{3}$ \\
$(6,6,6,6)$ & 27.00 & 37.80 & 13.34 & 19.18 & 28.06 & $A_{2}>A_{5}>A_{1}>A_{4}>A_{3}$ \\
$(10,1,1,1)$ & 29.45 & 41.52 & 15.29 & 21.46 & 29.89 & $A_{2}>A_{5}>A_{1}>A_{4}>A_{3}$ \\
$(10,10,1,1)$ & 27.77 & 36.63 & 13.95 & 19.62 & 27.49 & $A_{2}>A_{1}>A_{5}>A_{4}>A_{3}$ \\
$(10,10,10,1)$ & 27.04 & 30.94 & 12.34 & 16.30 & 23.70 & $A_{2}>A_{1}>A_{5}>A_{4}>A_{3}$ \\
$(14,15,1,1)$ & 26.79 & 32.35 & 9.60 & 18.47 & 25.12 & $A_{2}>A_{1}>A_{5}>A_{4}>A_{3}$ \\
$(16,17,18,19)$ & 26.70 & 31.51 & 9.61 & 18.52 & 24.59 & $A_{2}>A_{1}>A_{5}>A_{4}>A_{3}$ \\
$(20,20,20,20)$ & 26.72 & 31.17 & 9.63 & 18.56 & 24.38 & $A_{2}>A_{1}>A_{5}>A_{4}>A_{3}$ \\
\hline
\end{tabular}

Table 8. Ranking for different parameters of DGNNNWGBM.

\begin{tabular}{ccccccc}
\hline $\boldsymbol{R}$ & $S\left(\boldsymbol{A}_{\mathbf{1}}\right)$ & $\boldsymbol{S}\left(\boldsymbol{A}_{\mathbf{2}}\right)$ & $\boldsymbol{S}\left(\boldsymbol{A}_{\mathbf{3}}\right)$ & $\boldsymbol{S}\left(\boldsymbol{A}_{\mathbf{4}}\right)$ & $\boldsymbol{S}\left(\boldsymbol{A}_{\mathbf{5}}\right)$ & Ranking \\
\hline$(1,1,1,1)$ & 28.10 & 63.07 & 22.34 & 27.33 & 32.73 & $A_{2}>A_{5}>A_{4}>A_{1}>A_{3}$ \\
$(3,3,3,3)$ & 25.29 & 54.01 & 21.92 & 25.55 & 29.07 & $A_{2}>A_{5}>A_{4}>A_{1}>A_{3}$ \\
$(4,4,4,4)$ & 24.47 & 50.6 & 21.74 & 24.80 & 27.89 & $A_{2}>A_{5}>A_{4}>A_{1}>A_{3}$ \\
$(6,6,6,6)$ & 23.46 & 45.62 & 21.40 & 23.53 & 26.40 & $A_{2}>A_{5}>A_{4}>A_{1}>A_{3}$ \\
$(10,1,1,1)$ & 24.07 & 46.39 & 21.22 & 23.28 & 27.02 & $A_{2}>A_{5}>A_{1}>A_{4}>A_{3}$ \\
$(10,10,1,1)$ & 23.11 & 42.60 & 21.01 & 22.45 & 25.72 & $A_{2}>A_{5}>A_{1}>A_{4}>A_{3}$ \\
$(10,10,10,1)$ & 22.81 & 40.93 & 20.92 & 22.10 & 25.14 & $A_{2}>A_{5}>A_{1}>A_{4}>A_{3}$ \\
$(14,15,1,1)$ & 22.68 & 40.98 & 22.63 & 22.03 & 25.23 & $A_{2}>A_{5}>A_{1}>A_{3}>A_{4}$ \\
$(16,17,18,19)$ & 31.59 & 53.67 & 31.74 & 28.52 & 37.37 & $A_{2}>A_{5}>A_{3}>A_{1}>A_{4}$ \\
$(20,20,20,20)$ & 31.52 & 74.73 & 31.64 & 28.30 & 37.27 & $A_{2}>A_{5}>A_{3}>A_{1}>A_{4}$ \\
\hline
\end{tabular}

As shown in Table 7, when the parameter values are small, the ranking of the alternatives may be of little influence. When the parameter values are large, the ordering of $A_{1}$ and $A_{5}$ changes. However, the best alternative is the same, i.e., $A_{2}$. As shown in Table 8 , when the parameter values are small, the ranking of the alternatives may be of little influence, but when the parameter values are large, it has a great impact on the ranking results. Although the ranking changes greatly, the best alternative is still $A_{2}$. In practical applications, we usually take $R=(1,1, . ., 1)^{T}$, which is not only intuitive but also takes into account the effect of multiple parameters.

\subsection{Comparison Analysis}

In this section, we compare the DGNNNWBM and DGNNNWGBM operators proposed in this paper with the normal neutrosophic weighted Bonferroni mean (NNWBM) operator and normal neutrosophic weighted geometric Bonferroni mean (NNWGBM) operator proposed by Liu P and $\mathrm{Li} \mathrm{H} \mathrm{[6]} \mathrm{for} \mathrm{dealing} \mathrm{with} \mathrm{Example} \mathrm{5.1.} \mathrm{The} \mathrm{results} \mathrm{are} \mathrm{shown} \mathrm{in} \mathrm{Tables} \mathrm{8-14,} \mathrm{where} \mathrm{we} \mathrm{take} \mathrm{the} \mathrm{first}$ two values of the parameter $R$ in the DGNNNWBM and DGNNNWGBM operators as the parameter values $p, q$ in the NNWBM and NNWGBM operators.

According to the result, we conclude the following:

(1) From Tables 7 and 9, when $p, q$ take different values and the values are small, the NNWBM operator has three different ranking results, while the DGNNNWBM operator has only one. It shows that the stability of the DGNNNWBM operator is better than that of the NNWBM operator.

(2) From Tables 8 and 10, there is only one ranking result of the NNWGBM operaotr. However, Tables 11-14 show that when the parameter values $p, q$ are taken as $(10,10)$ and $(14,15)$, the result of the NNWBM operator is $T=0, I=1, F=1$, and the NNWGBM operator result is $T=1, I=0, F=0$. Regardless of whether the parameters $p, q$ change, the values of $a$ and $\delta$ in the NNWGBM operator are invariant. However, in this case, the DGNNNWBM and DGNNNWGBM 
operators consider more parameters, so they can overcome these problems that arise in the NNWBM and NNWGBM operators.

From this, we know that the NNWBM and NNWGBM operators lack stability and sensitivity. Compared to the NNWBM and NNWGBM operators, the DGNNNWBM and DGNNNWGBM are not only more general, but they are also more flexible.

Table 9. Liu and Li's method [6] (ranking for different parameters of the normal neutrosophic weighted Bonferroni mean (NNWBM)).

\begin{tabular}{ccccccc}
\hline$(p, q)$ & $S\left(A_{\mathbf{1}}\right)$ & $S\left(A_{\mathbf{2}}\right)$ & $S\left(A_{\mathbf{3}}\right)$ & $S\left(A_{\mathbf{4}}\right)$ & $S\left(A_{\mathbf{5}}\right)$ & Ranking \\
\hline$(1,1)$ & 69.31 & 126.23 & 24.29 & 52.83 & 72.66 & $A_{2}>A_{5}>A_{1}>A_{4}>A_{3}$ \\
$(3,3)$ & 78.69 & 209.45 & 57.12 & 77.09 & 108.89 & $A_{2}>A_{5}>A_{1}>A_{4}>A_{3}$ \\
$(4,4)$ & 70.23 & 200.1 & 54.65 & 74.78 & 102.21 & $A_{2}>A_{5}>A_{4}>A_{1}>A_{3}$ \\
$(6,6)$ & 58.8 & 182.3 & 44.67 & 68.65 & 88.42 & $A_{2}>A_{5}>A_{4}>A_{1}>A_{3}$ \\
$(10,1)$ & 67.93 & 127.63 & 43.67 & 80.84 & 74.68 & $A_{2}>A_{4}>A_{5}>A_{1}>A_{3}$ \\
\hline
\end{tabular}

Table 10. Liu and Li's method [6] (ranking for different parameters of the normal neutrosophic weighted geometric Bonferroni mean (NNWGBM)).

\begin{tabular}{ccccccc}
\hline$(p, q)$ & $S\left(A_{\mathbf{1}}\right)$ & $S\left(A_{\mathbf{2}}\right)$ & $S\left(A_{\mathbf{3}}\right)$ & $S\left(A_{\mathbf{4}}\right)$ & $S\left(A_{\mathbf{5}}\right)$ & Ranking \\
\hline$(1,1)$ & 62.89 & 149.2 & 14.17 & 54.77 & 70.31 & $A_{2}>A_{5}>A_{1}>A_{4}>A_{3}$ \\
$(3,3)$ & 61.89 & 146.55 & 13.78 & 53.1 & 68.49 & $A_{2}>A_{5}>A_{1}>A_{4}>A_{3}$ \\
$(4,4)$ & 61.6 & 145.91 & 13.66 & 52.71 & 68.02 & $A_{2}>A_{5}>A_{1}>A_{4}>A_{3}$ \\
$(6,6)$ & 61.24 & 145.12 & 13.78 & 52.23 & 67.45 & $A_{2}>A_{5}>A_{1}>A_{4}>A_{3}$ \\
$(10,1)$ & 58.03 & 128.07 & 9.32 & 49.42 & 61.20 & $A_{2}>A_{5}>A_{1}>A_{4}>A_{3}$ \\
\hline
\end{tabular}

Table 11. The DGNNNWBM operator and DGNNWGBM operator $R=(10,10,10,1)$.

\begin{tabular}{ccc}
\hline & DGNNNWBM & DGNNWGBM \\
\hline$A_{1}$ & $\langle(0.8053,0.0938),(0.6119,0.1447,0.1513)\rangle$ & $\langle(0.776,0.0785),(0.6137,0.6069,0.3126)\rangle$ \\
$A_{2}$ & $\langle(0.9374,0.0665),(0.5108,0.247,0.2609)\rangle$ & $\langle(0.8628,0.0289),(0.5141,0.7837,0.5514)\rangle$ \\
$A_{3}$ & $\langle(0.7006,0.1533),(0.3745,0.3875,0.2823)\rangle$ & $\langle(0.4772,0.0757),(0.3809,0.4822,0.3454)\rangle$ \\
$A_{4}$ & $\langle(0.6559,0.1119),(0.3997,0.3468,0.3391)\rangle$ & $\langle(0.6256,0.0615),(0.4053,0.6176,0.4645)\rangle$ \\
$A_{5}$ & $\langle(0.8808,0.0948),(0.5941,0.1819,0.272)\rangle$ & $\langle(0.8351,0.063),(0.5961,0.6959,0.3844)\rangle$ \\
\hline
\end{tabular}

Table 12. Liu and Li's method [6] (the NNWBM operator and NNWGBM operator $p=10, q=10$ ).

\begin{tabular}{ccc}
\hline & NNWBM & NNWGBM \\
\hline$A_{1}$ & $\langle(0.2491,0.0223),(0.252,0.6278,0.6089)\rangle$ & $\langle(0.938,0.0457),(1,0,0)\rangle$ \\
$A_{2}$ & $\langle(0.2608,0.0063),(0.3112,0.7388,0.6502)\rangle$ & $\langle(0.962,0.018),(1,0.2037,0)\rangle$ \\
$A_{3}$ & $\langle(0.159,0.0213),(0.1708,0.7887,0.6887)\rangle$ & $\langle(0.8029,0.2152),(0.7628,0.1948,0)\rangle$ \\
$A_{4}$ & $\langle(0.1913,0.011),(0.2205,0.8023,0.7488)\rangle$ & $\langle(0.8898,0.0494),(0.81,0.2168,0)\rangle$ \\
$A_{5}$ & $\langle(0.2574,0.0138),(0.3343,0.6173,0.7303)\rangle$ & $\langle(0.9555,0.0401),(1,0.1649,0)\rangle$ \\
\hline
\end{tabular}

Table 13. The DGNNNWBM operator and DGNNNWGBM operator $R=(14,15,1,1)$.

\begin{tabular}{ccc}
\hline & DGNNNWBM & DGNNNWGBM \\
\hline$A_{1}$ & $\langle(0.8131,0.0944),(0.6071,0.1287,0.1299)\rangle$ & $\langle(0.7755,0.0778),(0.611,0.6145,0.3321)\rangle$ \\
$A_{2}$ & $\langle(0.9493,0.0693),(0.4878,0.2317,0.2434)\rangle$ & $\langle(0.8611,0.0278),(0.4944,0.7966,0.5776)\rangle$ \\
$A_{3}$ & $\langle(0.7075,0.1552),(0.3562,0.364,0.2669)\rangle$ & $\langle(0.4607,0.0697),(0.3683,0.4855,0.3546)\rangle$ \\
$A_{4}$ & $\langle(0.6684,0.1148),(0.3741,0.3137,0.323)\rangle$ & $\langle(0.6253,0.0603),(0.3846,0.6332,0.4713)\rangle$ \\
$A_{5}$ & $\langle(0.8997,0.0995),(0.5762,0.1641,0.248)\rangle$ & $\langle(0.8345,0.062),(0.5809,0.7067,0.3868)\rangle$ \\
\hline
\end{tabular}


Table 14. Liu and Li's method [6] (the NNWBM operator and NNWGBM operator $p=14, q=15$ ).

\begin{tabular}{ccc}
\hline & NNWBM & NNWGBM \\
\hline$A_{1}$ & $\langle(0.2563,0.0275),(0,0.6164,0.5947)\rangle$ & $\langle(0.938,0.0457),(1,0,0)\rangle$ \\
$A_{2}$ & $\langle(0.2671,0.0077),(0.3202,1,0.6403)\rangle$ & $\langle(0.962,0.018),(1,0,0)\rangle$ \\
$A_{3}$ & $\langle(0.1638,0.0271),(0,1,0.6798)\rangle$ & $\langle(0.8029,0.2152),(1,0,0)\rangle$ \\
$A_{4}$ & $\langle(0.1955,0.0125),(0,1,1)\rangle$ & $\langle(0.8898,0.0494),(1,0,0)\rangle$ \\
$A_{5}$ & $\langle(0.2633,0.0168),(0.3418,0.6064,1)\rangle$ & $\langle(0.9555,0.0401),(1,0,0)\rangle$ \\
\hline
\end{tabular}

\section{Conclusions}

The multiple attribute decision-making method has a wide range of applications in many domains. The nonnegative normal neutrosophic number is more suitable for dealing with uncertain information, and the dual generalized weighted Bonferroni mean operator and dual generalized weighted geometric Bonferroni mean operator take into account the relationship between arbitrary aggregation arguments. Therefore, in this paper, the definition of nonnegative normal neutrosophic number has been proposed. The score function and accuracy function have been developed to overcome the deficiency, i.e., that the original function does not satisfy the ranking principle. Considering the connections between any two or more than two aggregation arguments, the dual generalized nonnegative normal neutrosophic weighted Bonferroni mean operator and dual generalized nonnegative normal neutrosophic weighted geometric Bonferroni mean operator were discussed. Meanwhile, some properties were investigated, such as idempotency, monotonicity, boundedness, and commutativity. Based on the dual generalized nonnegative normal neutrosophic weighted Bonferroni mean operator and dual generalized nonnegative normal neutrosophic weighted geometric Bonferroni mean operator, a method was developed to deal with a multiple attribute decision-making problem with nonnegative normal neutrosophic number. Further, we used the dual generalized nonnegative normal neutrosophic weighted Bonferroni mean and dual generalized nonnegative normal neutrosophic weighted geometric Bonferroni mean operators for aggregative information. Decision making obtain the satisfactory alternative according to actual need and preference by changing the values of $R$, which makes our proposed multiple attribute decision-making method more flexible and reliable. Further, compared with the method in Liu [6], our method shows that when the relationship between more aggregation arguments are considered, the aggregation result is more stable; when the parameter value is larger, the aggregation result is more sensitive.

Author Contributions: J.M. conceived, wrote and revised this paper; H.-L.H. provided ideas and suggestions for the revision of the paper.

Funding: This research was funded by National Natural Science Foundation Project, grant number (11701089); Fujian Natural Science Foundation, grant number (2018J01422).

Acknowledgments: This paper is supported by Institute of Meteorological Big Data-Digital Fujian and Fujian Key Laboratory of Data Science and Statistics.

Conflicts of Interest: The authors declare no conflict of interest.

\section{References}

1. Zadeh, L.A. Fuzzy sets. Inform. Contr. 1965, 8, 338-356. [CrossRef]

2. Atanassov, K.T. Intuitionistic fuzzy sets. Fuzzy. Set. Syst. 1986, 20, 87-96. [CrossRef]

3. Smarandache, F. A Unifying Field in Logics: Neutrosophy Logic; American Research Press: Rehoboth, DE, USA, 1999; pp. 1-141.

4. Yang, M.S.; Ko, C.H. On a class of fuzzy c-numbers clustering procedures for fuzzy data. Fuzzy Set. Syst. 1996, 84, 49-60. [CrossRef]

5. Wang, J.Q.; Li, K.J. Multi-criteria decision-making method based on induced intuitionistic normal fuzzy related aggregation operators. Int. J. Uncertain. Fuzziness Knowl. Base. Syst. 2012, 20, 559-578. [CrossRef]

6. Liu, P.; Li, H. Multiple attribute decision-making method based on some normal neutrosophic bonferroni mean operators. Neural Comput. Appl. 2017, 28,1-16. [CrossRef] 
7. Chatterjee, P.; Mondal, S.; Boral, S.; Banerjee, A.; Chakraborty, S. A novel hybrid method for non-traditional machining process selection using factor relationship and multi-attribute border approximation method. Facta Univ. Ser. Mech. Eng. 2017, 15, 439-456. [CrossRef]

8. Petković, D.; Madić, M.; Radovanović, M.; Gečevska, V. Application of the performance selection index method for colving machining MCDM problems. Facta Univ. Ser. Mech. Eng. 2017, 15, 97-106. [CrossRef]

9. Roy, J.; Adhikary, K.; Kar, S.; Pamučar, D. A rough strength relational DEMATEL model for analysing the key success factors of hospital service quality. Decis. Mak. Appl. Manag. Eng. 2018, 1, 121-142. [CrossRef]

10. Badi, I.A.; Abdulshahed, A.M.; Shetwan, A. A case study of supplier selection for a steelmaking company in Libya by using the Combinative Distance-based ASsessment (CODAS) model. Decis. Mak. Appl. Manag. Eng. 2018, 1, 1-12. [CrossRef]

11. Lee, S.H.; Ryu, K.H.; Sohn, G. Study on entropy and similarity measure for fuzzy set. IEICE Trans. Inform. Syst. 2009, 92, 1783-1786. [CrossRef]

12. Nan, J.X.; Wang, T.; An, J.J. Intuitionistic fuzzy distance based TOPSIS method and application to MADM. Int. J. Fuzzy Syst. Appl. 2017, 5, 43-56. [CrossRef]

13. Joshi, R.; Kumar, S. A new parametric intuitionistic fuzzy entropy and its applications in multiple attribute decision making. Int. J. Appl. Comput. Sci. Math. 2018, 4, 52. [CrossRef]

14. Liu. M.; Ren, H. A new intuitionistic fuzzy entropy and application in multi-attribute decision making. Information 2014, 5, 587-601. [CrossRef]

15. Atanassov, K.; Gargov, G. Interval-valued intuitionistic fuzzy sets. Fuzzy Set. Syst. 1989, 31, $343-349$. [CrossRef]

16. Huang, H.L. (T, S)-based interval-valued intuitionistic fuzzy composition matrix and its application for clustering. Iranian J. Fuzzy Syst. 2012, 9, 7-19.

17. Chen, S.M.; Huang, Z.C. Multiattribute decision making based on interval-valued intuitionistic fuzzy values and linear programming methodology. Inform. Sci. 2017, 381, 341-351. [CrossRef]

18. Biswas, A.; Kumar, S. An integrated TOPSIS approach to MADM with interval-valued intuitionistic fuzzy settings. Adv. Comput. Commun. Paradig. 2018, 706, 533-543.

19. Peng, J.J.; Wang, J.Q.; Zhang, H.Y. An outranking approach for multi-criteria decision-making problems with simplified neutrosophic sets. Appl. Soft Comput. 2014, 25, 336-346. [CrossRef]

20. Bausys, R.; Zavadskas, E.K.; Kaklauskas, A. Application of Neutrosophic Set to Multicriteria Decision Making by COPRAS. Econ. Comput. Econ. Cybern. Stud. Res. 2015, 49, 91-106.

21. Majumdarar, P.; Samant, S.K. On similarity and entropy of neutrosophic sets. J. Intell. Fuzzy Syst. 2014, $26,1245-1252$.

22. Wang, H.; Smarandache, F.; Zhang,Y.Q.; Sunderraman, R. Interval Neutrosophic Sets and Logic: Theory and Applications in Computing; Hexis: Corona, CA, USA, 2005.

23. Wang, H.; Smarandache, F.; Zhang, Y.Q.; Sunderraman, R. Single valued neutrosophic sets. Multisp. Multistruct. 2010, 4, 410-413.

24. Zhang, H.Y.; Wang, J.Q.; Chen, X.H. An outranking approach for multi-criteria decision-making problems with interval-valued neutrosophic sets. Neural Comput. Appl. 2016, 27, 615-627. [CrossRef]

25. Zhang, H.Y.; Ji, P.; Wang, J.Q.; Chen, X.H. An improved weighted correlation coefficient based on integrated weight for interval neutrosophic sets and its application in multi-criteria decision-making problems. Int. J. Comput. Intell. Syst. 2015, 8, 1027-1043. [CrossRef]

26. Tian, Z.P.; Zhang, H.Y.; Wang, J.; Wang, J.Q.; Chen, X.H. Multi-criteria decision-making method based on a cross-entropy with interval neutrosophic sets. Int. J. Syst. Sci. 2016, 47, 3598-3608. [CrossRef]

27. Huang, H.L. New distance measure of single-valued neutrosophic sets and its application. Int. J. Intell. Syst. 2016, 31, 1021-1032. [CrossRef]

28. Ye, J. Single valued neutrosophic cross-entropy for multicriteria decision making problems. Appl. Math. Model. 2014, 38, 1170-1175. [CrossRef]

29. Zhang, H.; Zheng, Q.; Liu, T.; Qu, Y. Mixed intuitionistic fuzzy aggregation operators decreasing results of unusual IFNs. In Proceedings of the IEEE International Conference on Fuzzy Systems (FUZZ-IEEE), Vancouver, BC, Canada, 24-29 July 2016, 896-903.

30. Sirbiladze, G.; Sikharulidze, A. Extensions of probability intuitionistic fuzzy aggregation operators in fuzzy MCDM/MADM. Int. J. Inform. Tech. Decis. Mak. 2018, 17, 621-655. [CrossRef] 
31. Wang, W.; Liu, X. Intuitionistic fuzzy information aggregation using einstein operations. IEEE Trans. Fuzzy Syst. 2012, 20, 923-938. [CrossRef]

32. Wang, J.Q.; Li, K.J.; Zhang, H.Y.; Chen, X.H. A score function based on relative entropy and its application in intuitionistic normal fuzzy multiple criteria decision making. J. Intell. Fuzzy Syst. 2013, 25, 567-576.

33. Wang, J.Q.; Li, K.J. Multi-criteria decision-making method based on intuitionistic normal fuzzy aggregation operators. Syst. Eng. Theor. Pract. 2013, 33, 1501-1508.

34. Wang, J.Q.; Zhou, P.; Li, K.J.; Zhang, H.Y.; Chen, X.H. Multi-criteria decision-making method based on normal intuitionistic fuzzy induced generalized aggregation operator. Top 2014, 22, 1103-1122. [CrossRef]

35. Peng, J.J.; Wang, J.Q.; Wu, X.H. Multi-valued neutrosophic sets and power aggregation operators with their applications in multi-criteria group decision-making problems. Int. J. Comput. Intell. Syst. 2015, 8, 345-363. [CrossRef]

36. Liu, P.D.; Liu, X. The neutrosophic number generalized weighted power averaging operator and its application in multiple attribute group decision making. Int. J. Mach. Learn. Cybern. 2018, 9, 347-358. [CrossRef]

37. Ye, J. Multiple attribute decision-making method based on the possibility degree ranking method and ordered weighted aggregation operators of interval neutrosophic numbers. J. Intell. Fuzzy Syst. 2015, $28,1307-1317$.

38. Wang, R.; Li, Y.L. Generalized single-valued neutrosophic hesitant fuzzy prioritized aggregation operators and their applications to multiple criteria decision-making. Information 2018, 9, 10. [CrossRef]

39. Liu, P.D.; Teng, F. Multiple attribute decision making method based on normal neutrosophic generalized weighted power averaging operator. Int. J. Mach. Learn. Cybern. 2018, 9, 281-293. [CrossRef]

40. Liu, P.D.; Teng, F. Multiple attribute group decision making methods based on some normal neutrosophic number heronian mean operators. J. Intell. Fuzzy Syst. 2017, 32, 2375-2391. [CrossRef]

41. Liu, P.D.; Wang, P.; Liu, J.L. Normal neutrosophic frank aggregation operators and their application in multi-attribute group decision making. Int. J. Mach. Learn. Cybern. 2017, 1, 1-20. [CrossRef]

42. Şahin, R. Normal neutrosophic multiple attribute decision making based on generalized prioritized aggregation operators. Neural. Comput. Appl. 2017,1-21. [CrossRef]

43. Bonferroni, C. Sulle medie multiple di potenze. Bollettino dell'Unione Matematica Italiana 1950, 5, $267-270$.

44. Zhang, R.T.; Wang, J.; Zhu, X.M.; Xia, M.; Yu, M. Some generalized pythagorean fuzzy bonferroni mean aggregation operators with their application to multiattribute group decision-making. Complexity 2017, 6, 1-16. [CrossRef]

(C) 2018 by the authors. Licensee MDPI, Basel, Switzerland. This article is an open access article distributed under the terms and conditions of the Creative Commons Attribution (CC BY) license (http:/ / creativecommons.org/licenses/by/4.0/). 